\title{
Research Article \\ Dynamics of Mutualism-Competition-Predator System with Beddington-DeAngelis Functional Responses and Impulsive Perturbations
}

\author{
Xiaoming Fan, ${ }^{1}$ Zhigang Wang, $^{1,2}$ and Fuquan Jiang ${ }^{3}$ \\ ${ }^{1}$ School of Mathematics Science, Harbin Normal University, Harbin 150500, China \\ ${ }^{2}$ School of Mathematics and Statistics, Northeast Normal University, Changchun 130024, China \\ ${ }^{3}$ Foundation Department, Harbin Finance University, Harbin 150030, China \\ Correspondence should be addressed to Zhigang Wang,wangzg2003205@yahoo.com.cn
}

Received 17 March 2012; Revised 10 May 2012; Accepted 14 May 2012

Academic Editor: Ferhan M. Atici

Copyright (C) 2012 Xiaoming Fan et al. This is an open access article distributed under the Creative Commons Attribution License, which permits unrestricted use, distribution, and reproduction in any medium, provided the original work is properly cited.

We explore the dynamics of a class of mutualism-competition-predator interaction models with Beddington-DeAngelis functional responses and impulsive perturbations. Sufficient conditions for existence of positive periodic solution are established by using a continuation theorem in coincidence degree theory, which have been extensively applied in studying existence problems in differential equations and difference equations. In addition, sufficient criteria are given for the global stability and the globally exponential stability of system by employing comparison principle and Lyapunov method.

\section{Introduction}

The ecological predator-prey systems and impulsive functional differential equations have been studied extensively by many authors [1-7]. A predator's per capita feeding rate on prey, or its functional response, provides a foundation for predator-prey theory. Since 1959, Holling's prey-dependent type II functional response, a model that is a function of prey abundance only, has served as the basis for a large literature on predator-prey theory. The traditional Kolmogorov type predator-prey model with Holling's type II functional response:

$$
\mathbf{x}^{\prime}=r \mathbf{x}\left(1-\frac{\mathbf{x}}{K}\right)-c \mathbf{y}\left(\frac{\mathbf{x}}{m+\mathbf{x}}\right), \quad \mathbf{y}^{\prime}=-d \mathbf{y}+f \mathbf{y}\left(\frac{\mathbf{x}}{m+\mathbf{x}}\right)
$$


and its various generalized forms have received great attention from both theoretical and mathematical biologists and have been well studied [1, 8, 9]. In (1.1), $\mathbf{x}$ and $\mathbf{y}$ represent the densities of prey specie and predator specie at time $t$, respectively. Predator-prey model with Holling's type II functional response assumes that predators do not interfere with one another's activities; thus competition among predators for food occurs only via the depletion of prey. However, when predators have to search for food (and therefore, have to share or compete for food), the functional response in a prey-predator model should be predatordependent. The predator-prey system with the Beddington-DeAngelis functional response:

$$
\mathbf{x}^{\prime}=r \mathbf{x}\left(1-\frac{\mathbf{x}}{K}\right)-\frac{\alpha \mathbf{x y}}{a+b \mathbf{x}+c \mathbf{y}}, \quad \mathbf{y}^{\prime}=-d \mathbf{y}+\frac{\beta \mathbf{x y}}{a+b \mathbf{x}+c \mathbf{y}}
$$

was originally introduced by Beddington [10] and DeAngelis et al. [11], independently, where $\mathbf{x}$ and $\mathbf{y}$ represent the densities of prey specie and predator specie at time $t$, respectively. The main difference of this functional response from Holling's type II functional response is that it contains an extra term presenting mutual interference by predators. In this model, individuals from a population of two or more predators not only allocate time to searching for and processing prey, but also spend some time engaging in encounters with other predators, the Beddington-DeAngelis type can provide better descriptions of predator feeding over a range of predator-prey abundances.

On the other hand, differential equations with impulsive effects form a wide set of different problems. During the last three decades those problems were intensively studied. Some authors devote themselves to the study of impulsive differential equation [12-22]. The main definitions and results of the theory of systems of ordinary differential equations with impulse effects were given in $[12,13,15]$. Similarity and differentiality of such problems of applied mathematics with corresponding problems of ordinary differential equations (and without the conditions of impulsive effects) were demonstrated, and general characteristics of these systems were described. Periodic and almost-periodic solutions of differential equations with impulsive effects were studied in [18]. De La Sen investigated time-varying systems with nonnecessarily bounded everywhere continuous time-differentiable timevarying point delays [19]. The delay-free and delayed dynamics are assumed to be timevarying and impulsive, the constructed solution trajectories of both the unforced and forced systems are obtained from different (input-state space/output space and state space to output space) operators. The system stability and the compactness of the operators describing the solution trajectories are well investigated. De La Sen and Luo also obtain sufficiency-type stability results for time-delay linear systems with constant point delays under impulsive inputs of impulses of state-dependent amplitudes occurring separately through time. They proved that the amplitudes of the impulses and the time intervals between impulses may be chosen sufficiently large if the delay-free dynamics is sufficiently stable compared to the delayed one [20]. Xu and Sun investigated the problem of finite-time stability of linear time-varying singular systems with impulses at fixed times, they have proposed a sufficient condition for linear singular impulsive systems to be finite-time stable in terms of a set of coupled matrix inequalities [21]. Zhang and Sun also considered the stability of impulsive linear differential equations with time delay. By using Lyapunov functions and analysis technique, they get some results for the stability of impulsive linear differential equations with time delay [22]. 
Some impulsive factors have also great impact on the growth of a population. For example, we notice that the births of many species are not continuous but happen at some regular time (e.g., the births of some wildlife are seasonal). Moreover, the human beings have been harvesting or stocking species at some time, then the species is affected by another type of impulse. If we incorporate these impulsive factors into the models of population interactions, the models must be governed by impulsive ordinary differential equations.

Population communities are embedded in periodically varying environments. Therefore, this study should take into account the biological and environmental periodicity (e.g., seasonal effects of weather, food supplies, and mating habits), we focus on the existence of periodic solution with strictly positive components. In real world, any biological or environmental parameters are naturally subject to fluctuation in time, so it is reasonable to study the corresponding nonautonomous system.

The first author investigated the existence of positive periodic solutions of a nonautonomous competitive Lotka-Volterra system with impulse and Holling type III functional response [23]. An $N$-dimensional Lotka-Volterra system with fixed moments of impulsive perturbations is given in [24] by Ahmad and Stamova. By means of piecewise continuous functions which are modifications of classical Lyapunov' functions they give sufficient conditions for asymptotic stability of the solutions. Inspired by [23, 24], in this paper, we focus our attention on the existence of periodic solution and globally asymptotic stability of solutions for multispecies mutualism-competition-predator system with impulses in which the competition among predator species and the mutualism among prey species are simultaneously considered. In general, mutualism is relevant to two species [25, 26], thus we focus on two prey species and assume that there exists the relation of mutualism between two prey species. The primary approach is based on the coincidence degree and its related continuation theorem [27], which has been widely used in dealing with the existence of periodic solutions of differential equations. We will investigate the following nonautonomous system:

$$
\begin{array}{r}
\dot{x}_{1}(t)=x_{1}(t)\left(b_{1}(t)-a_{1}(t) x_{1}(t)-\frac{x_{1}(t)}{\mu_{1}(t)+\omega_{1}(t) x_{2}(t)}\right. \\
\left.-\sum_{k=1}^{n} \frac{c_{1 k}(t) y_{k}(t)}{\alpha_{1 k}(t)+\beta_{1 k}(t) x_{1}(t)+\gamma_{1 k}(t) y_{k}(t)}\right), \\
\dot{x}_{2}(t)=x_{2}(t)\left(b_{2}(t)-a_{2}(t) x_{2}(t)-\frac{x_{2}(t)}{\mu_{2}(t)+\omega_{2}(t) x_{1}(t)}\right. \\
\dot{y}_{j}(t)=y_{j}(t)\left(-\sum_{k=1}^{n} \frac{c_{2 k}(t) y_{k}(t)}{\alpha_{2 k}(t)+\beta_{2 k}(t) x_{2}(t)+\gamma_{2 k}(t) y_{k}(t)}\right), \\
\left.-\sum_{k=1}^{n} \delta_{j k}(t) y_{k}(t)\right),
\end{array}
$$




$$
\begin{gathered}
t \neq t_{k}, \quad\left(k \in \mathbb{Z}^{+}\right), \quad j=1, \ldots, n, \\
\Delta x_{i}(t)=x_{i}\left(t^{+}\right)-x_{i}\left(t^{-}\right)=b_{i k} x_{i}(t), \\
\Delta y_{j}(t)=y_{j}\left(t^{+}\right)-y_{j}\left(t^{-}\right)=\widetilde{b}_{j k} y_{j}(t), \\
t=t_{k}, \quad\left(k \in \mathbb{Z}^{+}\right), \quad i=1,2, \quad j=1, \ldots, n,
\end{gathered}
$$

where

(i) $x_{i}(t)(i=1,2)$ denote the densities of prey species at time $t$, respectively;

(ii) $y_{j}(t)(j=1, \ldots, n)$ denote the density of predator species at time $t$, respectively;

(iii) $b_{i k}$ represent the sum of the birth rate and the harvesting (or stocking) rate of $x_{i}(t)$ at time $t$, respectively;

(iv) $\tilde{b}_{j k}$ represent the sum of the birth rate and the harvesting (or stocking) rate of $y_{j}(t)$ at time $t$, respectively;

(v) $x_{i}\left(t^{+}\right)$and $x_{i}\left(t^{-}\right)$represent the right and left limit of $x_{i}(t)$ at $t, y_{j}\left(t^{+}\right)$and $y_{j}\left(t^{-}\right)$ represent the right and left limit of $y_{j}(t)$ at $t$.

Let $g(t)$ be a bounded continuous function on $\mathbb{R}$. Define

$$
g^{\ell}=\inf _{t \in \mathbb{R}} g(t), \quad g^{u}=\sup _{t \in \mathbb{R}} g(t)
$$

Particularly, if $g(t)$ are $T$-periodic functions with respect to $t$, we denote

$$
\bar{g}=\frac{1}{T} \int_{0}^{T} g(t) d t
$$

The range of the indices $i \in\{1,2\}$ and $j, k \in\{1, \ldots, n\}$ are used in this paper unless otherwise stated.

Throughout the paper, we give the hypotheses as follows

(A1) for any $t \in \mathbb{R}, b_{i}(t), a_{i}(t), r_{j}(t), \mu_{i}(t), \omega_{i}(t), d_{j i}(t), \delta_{j k}(t), c_{i k}(t), \alpha_{i k}, \beta_{i k}, \gamma_{i k}$ are nonnegative continuous $T$-periodic functions and $\alpha_{i j}=\alpha_{j i}, \beta_{i j}=\beta_{j i}, \gamma_{i j}=\gamma_{j i}$;

(A2) $b_{i k}, \tilde{b}_{j k}>0,1+b_{i k}>0,1+\tilde{b}_{j k}>0, b_{i k}, \tilde{b}_{j k}\left(k \in \mathbb{Z}^{+}\right)$are constants. There exists a positive integer $q$, such that $t_{k+q}=t_{k}+T, b_{i(k+q)}=b_{i k}, \widetilde{b}_{j(k+q)}=\tilde{b}_{j k},\left(k \in \mathbb{Z}^{+}\right)$. Without loss of generality, we also suppose that $t_{k} \neq 0$ and $[0, T] \cap\left\{t_{k}: k \in \mathbb{Z}^{+}\right\}=\left\{t_{1}, t_{2}, \ldots, t_{s}\right\}$, then it follows that $q=s$;

(A3) $x_{i}(t), y_{j}(t)$ is left-continuous at $t_{k}$, that is, the following relations are satisfied:

$$
\begin{aligned}
& x_{i}\left(t_{k}^{-}\right)=x_{i}\left(t_{k}\right), \quad x_{i}\left(t_{k}^{+}\right)=\left(1+b_{i k}\right) x_{i}\left(t_{k}\right), \quad k \in \mathbb{Z}^{+}, \\
& y_{j}\left(t_{k}^{-}\right)=y_{j}\left(t_{k}\right), \quad y_{j}\left(t_{k}^{+}\right)=\left(1+\tilde{b}_{j k}\right) y_{j}\left(t_{k}\right), \quad k \in \mathbb{Z}^{+} ;
\end{aligned}
$$


(A4) $t_{1}<t_{2}<\cdots$ and $\lim _{k \rightarrow \infty} t_{k}=\infty$;

(A5) $x_{i}\left(t_{0}^{+}\right)>0, y_{j}\left(t_{0}^{+}\right)>0$.

A brief description of the organization of this paper is as follows. The basic concepts and lemmas are given in Section 2. The main results in this paper are stated in Theorems 3.1, 4.5, and 4.6.

\section{Basic Concepts and Lemma}

Let $\varphi:[0, T] \subset J \rightarrow \mathbb{R}$ be a piecewise continuous function with points of discontinuity $t_{1}, \ldots, t_{s}$, we denote

$$
P C_{T}=\{\varphi: \varphi(0)=\varphi(T)\}, \quad P C_{T}^{1}=\left\{\varphi \in P C_{T}:\left(\frac{d \varphi}{d t}\right) \in P C_{T}\right\}
$$

Suppose

$$
\|\varphi\|_{P C_{T}}=\sup _{t \in[0, T]}|\varphi(t)|, \quad\|\varphi\|_{P C_{T}^{1}}=\max \left\{\|\varphi\|_{P C_{T}}\|\dot{\varphi}\|_{P C_{T}}\right\} .
$$

It can be easily proved that $P C_{T}$ and $P C_{T}^{1}$ are Banach spaces under the condition endowed the above norms.

Make the change of variables $x_{i}(t)=\exp \left\{u_{i}(t)\right\}, y_{j}(t)=\exp \left\{v_{j}(t)\right\}$, then (1.1) can be reformulated as

$$
\begin{aligned}
\dot{u}_{1}(t)= & b_{1}(t)-a_{1}(t) \exp \left\{u_{1}(t)\right\}-\frac{\exp \left\{u_{1}(t)\right\}}{\mu_{1}(t)+\omega_{1}(t) \exp \left\{u_{2}(t)\right\}} \\
& -\sum_{k=1}^{n} \frac{c_{1 k}(t) \exp \left\{v_{k}(t)\right\}}{\alpha_{1 k}(t)+\beta_{1 k}(t) \exp \left\{u_{1}(t)\right\}+\gamma_{1 k}(t) \exp \left\{v_{k}(t)\right\}}, \\
\dot{u}_{2}(t)= & b_{2}(t)-a_{2}(t) \exp \left\{u_{2}(t)\right\}-\frac{\exp \left\{u_{2}(t)\right\}}{\mu_{1}(t)+\omega_{1}(t) \exp \left\{u_{1}(t)\right\}} \\
& -\sum_{k=1}^{n} \frac{c_{2 k}(t) \exp \left\{v_{k}(t)\right\}}{\alpha_{2 k}(t)+\beta_{2 k}(t) \exp \left\{u_{2}(t)\right\}+\gamma_{2 k}(t) \exp \left\{v_{k}(t)\right\}}, \\
\dot{y}_{j}(t)= & -r_{j}(t)+\sum_{k=1}^{2} \frac{d_{j k}(t) \exp \left\{u_{k}(t)\right\}}{\alpha_{j k}(t)+\beta_{j k}(t) \exp \left\{u_{k}(t)\right\}+\gamma_{j k}(t) \exp \left\{v_{j}(t)\right\}} \\
& -\sum_{k=1}^{n} \delta_{j k}(t) \exp \left\{v_{k}(t)\right\},
\end{aligned}
$$




$$
\begin{gathered}
t \neq t_{k}, \quad\left(k \in \mathbb{Z}^{+}\right), \quad j=1, \ldots, n, \\
\Delta u_{i}(t)=u_{i}\left(t^{+}\right)-u_{i}\left(t^{-}\right)=\ln \left(1+b_{i k}\right), \\
\Delta v_{j}(t)=v_{j}\left(t^{+}\right)-v_{j}\left(t^{-}\right)=\ln \left(1+\tilde{b}_{j k}\right), \\
t=t_{k} \quad\left(k \in \mathbb{Z}^{+}\right), \quad i=1,2, \quad j=1, \ldots, n .
\end{gathered}
$$

For (1.3) and (2.3), we have similar lemma and definitions. So we only relate such results for (1.3).

Definition 2.1. The mapping $\mathbf{x}:[0, T] \rightarrow \mathbb{R}^{n+2}, \mathbf{x}(t)=\left(x_{1}(t), x_{2}(t), y_{1}(t), \ldots, y_{n}(t)\right)$ is called a solution of system $(1.1)$ in $[0, T]$, if

(1) $\mathbf{x}(t)$ is piecewise continuous in $[0, T]$ and $[0, T] \cap\left\{t_{k}: k \in \mathbb{Z}^{+}\right\}$are discontinuous points of the first kind of $\mathbf{x}(t)$, and $\mathbf{x}(t)$ are left continuous at $[0, T] \cap\left\{t_{k}: k \in \mathbb{Z}^{+}\right\}$;

(2) $\mathbf{x}(t)$ satisfies system in $[0, T]$.

Definition 2.2. The mapping $\mathbf{x}: J \rightarrow \mathbb{R}^{n+2}$ is called a $T$-periodic solution of system (1.1) if

(1) $\mathbf{x}(t)=\left(x_{1}(t), x_{2}(t), y_{1}(t), \ldots, y_{n}(t)\right)$ is a solution of system (1.1);

(2) $\mathbf{x}(t)$ satisfies $\mathbf{x}(t+T-0)=\mathbf{x}(t-0), t \in \mathbb{R}$.

Obviously, if $\mathbf{x}(t)$ is a solution of (1.1) satisfying $\mathbf{x}(0)=\mathbf{x}(T)$ in $[0, T]$, then from the periodicity of the vector field of (1.1), we know that

$$
\mathbf{x}^{*}(t)= \begin{cases}\mathbf{x}(t-\ell T), & t \in[\ell T,(\ell+1) T] \backslash\left\{t_{k}: k \in \mathbb{Z}^{+}\right\}, \ell \in \mathbb{N}, \\ \mathbf{x}^{*}, & (t) \text { is left countious at } t_{k}\end{cases}
$$

is an T-periodic solution for (1.1). Thus, the problem which discusses the existence of solution of (1.1) will be transformed to discuss the existence of periodic solution for (1.1) in $[0, T]$ satisfying $\mathbf{x}(0)=\mathbf{x}(T)$. In order to explore the existence of positive periodic solutions of (1.1), we recall some concepts and results on coincidence degree from [27, pages 39-40], borrowing notations there.

Let $\mathbb{X}, \mathbb{Z}$ be normed vector spaces, $\mathcal{L}:$ Dom $\mathcal{L} \subseteq \mathbb{X} \rightarrow \mathbb{Z}$ a linear mapping, and $\mathcal{N}$ : $\mathbb{X} \rightarrow \mathbb{Z}$ a continuous mapping. If dimker $\mathcal{L}=$ comdim $\operatorname{Im} \mathcal{L}<+\infty$ and $\operatorname{Im} \mathcal{L}$ is a closed in $\mathbb{Z}$, then the mapping $\mathcal{L}$ will be called a Fredholm mapping of index zero. If $\mathcal{L}$ is a Fredholm mapping of index zero, there exists continuous projects $D: \mathbb{X} \rightarrow \mathbb{X}$ and $Q: \mathbb{Z} \rightarrow \mathbb{Z}$ such that $\operatorname{Im} p=\operatorname{Ker} \mathcal{L}, \operatorname{Im} \mathcal{L}=\operatorname{Ker} \mathcal{Q}=\operatorname{Im}(I-\mathcal{Q})$. It follows that $\left.\mathcal{L}\right|_{\text {Dom } \mathcal{L} \operatorname{Ker}} p:(I-D) \mathbb{X} \rightarrow \operatorname{Im} \mathcal{L}$ has an inverse which is denoted by $K_{p}$. If $\Omega$ be an open bounded subset of $\mathbb{X}$, the mapping $N$ will be called $\mathcal{L}$-compact on $\bar{\Omega}$ provided that $\mathcal{Q} \mathcal{N}(\bar{\Omega})$ is bounded, and $K_{p}(I-Q) \mathcal{N}: \bar{\Omega} \rightarrow \mathbb{X}$ is compact. Since $\operatorname{Im} Q$ is isomorphic to $\operatorname{Ker} \mathcal{L}$, there exists an isomorphism $\mathcal{\partial}: \operatorname{Im} Q \rightarrow \operatorname{Ker} \mathcal{L}$.

The following continuation theorem is due to Gaines and Mawhin [27].

Lemma 2.3 (Continuation Theorem in [27]). Let $\perp$ a Fredholm mapping of index zero and $\mathcal{N}$ be $\mathcal{L}$-compact on $\bar{\Omega}$. Suppose that 
(a) for each $\lambda \in(0,1)$, every solution $\mathbf{x}$ of $\mathcal{L} \mathbf{x}=\lambda \mathcal{N} \mathbf{x}$ such that $\mathbf{x} \notin \partial \Omega$;

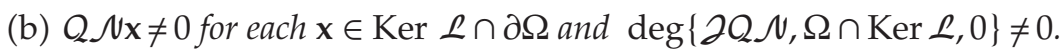

Then the operator equation $\mathcal{L} \mathbf{x}=\mathcal{N}$ has at least one solution lying in $\operatorname{Dom} \mathcal{\perp} \cap \bar{\Omega}$.

We can show that the solution of (1.1) with positive initial value remains positive too, that is, the following Lemma 2.4 holds.

Lemma 2.4. Suppose the hypotheses (A1)-(A5) hold. $x_{i}(t), y_{j}(t)$ is a solution of (1.1), then

$$
x_{i}(t), y_{j}(t)>0, \quad t \in J
$$

Proof. By integrating of (1.1) in the interval $\left(t_{0}, t_{1}\right]$, we have

$$
\begin{gathered}
x_{1}(t)=x_{i}\left(t_{0}^{+}\right) \exp \left\{\int _ { t _ { 0 } } ^ { t } \left(b_{1}(t)-a_{1}(t) x_{1}(t)-\frac{x_{1}(t)}{\mu_{1}(t)+\omega_{1}(t) x_{2}(t)}\right.\right. \\
\left.\left.-\sum_{k=1}^{n} \frac{c_{1 k}(t) y_{k}(t)}{\alpha_{1 k}(t)+\beta_{1 k}(t) x_{1}(t)+\gamma_{1 k}(t) y_{k}(t)}\right) d t\right\}, \\
x_{2}(t)=x_{i}\left(t_{0}^{+}\right) \exp \left\{\int _ { t _ { 0 } } ^ { t } \left(b_{2}(t)-a_{2}(t) x_{2}(t)-\frac{x_{2}(t)}{\mu_{2}(t)+\omega_{2}(t) x_{1}(t)}\right.\right. \\
y_{j}(t)=y_{j}\left(t_{0}^{+}\right) \exp \left\{\int_{t_{0}}^{t}\left(-\sum_{k=1}^{n} \frac{c_{2 k}(t) y_{k}(t)+\sum_{k=1}^{2} \frac{d_{j k}(t) x_{k}(t)}{\alpha_{j k}(t)+\beta_{j k}(t) x_{k}(t)+\gamma_{j k}(t) y_{j}(t)}}{\alpha_{2 k}(t) x_{2}(t)+\gamma_{2 k}(t) y_{k}(t)}\right) d t,\right. \\
\left.\left.-\sum_{k=1}^{n} \delta_{j k}(t) y_{k}(t)\right) d t\right\},
\end{gathered}
$$

for $t \in\left(t_{0}, t_{1}\right]$.

There does not exist point of discontinuity of $x_{i}(t), y_{j}(t)$ in the interval $\left(t_{0}, t_{1}\right]$, it is obvious that $x_{i}(t)>0, y_{j}(t)>0$ for $t \in\left(t_{0}, t_{1}\right]$, hence $\mathbf{x}\left(t_{1}\right)>0$. We have from (1.1) that

$$
x_{i}\left(t_{1}^{+}\right)=\left(1+b_{i k}\right) x_{i}\left(t_{1}\right), \quad y_{j}\left(t_{1}^{+}\right)=\left(1+\widetilde{b}_{j k}\right) y_{j}\left(t_{1}\right)
$$

It follows from (A2) that

$$
x_{i}\left(t_{1}^{+}\right)=\left(1+b_{i k}\right) x_{i}\left(t_{1}\right)>0, \quad y_{j}\left(t_{1}^{+}\right)=\left(1+\tilde{b}_{j k}\right) y_{j}\left(t_{1}\right)>0
$$


We now integrate (1.1) in the interval $\left(t_{1}, t_{2}\right]$, and we have

$$
\begin{gathered}
x_{1}(t)=x_{i}\left(t_{1}^{+}\right) \exp \left\{\int _ { t _ { 0 } } ^ { t } \left(b_{1}(t)-a_{1}(t) x_{1}(t)-\frac{x_{1}(t)}{\mu_{1}(t)+\omega_{1}(t) x_{2}(t)}\right.\right. \\
\left.\left.-\sum_{k=1}^{n} \frac{c_{1 k}(t) y_{k}(t)}{\alpha_{1 k}(t)+\beta_{1 k}(t) x_{1}(t)+\gamma_{1 k}(t) y_{k}(t)}\right) d t\right\}, \\
x_{2}(t)=x_{i}\left(t_{1}^{+}\right) \exp \left\{\int _ { t _ { 0 } } ^ { t } \left(b_{2}(t)-a_{2}(t) x_{2}(t)-\frac{x_{2}(t)}{\mu_{2}(t)+\omega_{2}(t) x_{1}(t)}\right.\right. \\
y_{j}(t)=y_{j}\left(t_{1}^{+}\right) \exp \left\{\int_{t_{0}}^{t}\left(-\sum_{k=1}^{n} \frac{c_{2 k}(t) y_{k}(t)}{\alpha_{2 k}(t)+\beta_{2 k}(t) x_{2}(t)+\gamma_{2 k}(t) y_{k}(t)}\right) d t\right\}, \\
\left.\left.-\sum_{k=1}^{n} \delta_{j k}(t) y_{k}(t)\right) d t\right\},
\end{gathered}
$$

for $t \in\left(t_{1}, t_{2}\right]$. From the above relation it follows that $x_{i}(t)>0$ for $t \in\left(t_{1}, t_{2}\right]$.

By similar arguments, we can obtain that

$$
\begin{gathered}
x_{1}(t)=x_{i}\left(t_{k}^{+}\right) \exp \left\{\int _ { t _ { 0 } } ^ { t } \left(b_{1}(t)-a_{1}(t) x_{1}(t)-\frac{x_{1}(t)}{\mu_{1}(t)+\omega_{1}(t) x_{2}(t)}\right.\right. \\
\left.\left.-\sum_{k=1}^{n} \frac{c_{1 k}(t) y_{k}(t)}{\alpha_{1 k}(t)+\beta_{1 k}(t) x_{1}(t)+\gamma_{1 k}(t) y_{k}(t)}\right) d t\right\}, \\
x_{2}(t)=x_{i}\left(t_{k}^{+}\right) \exp \left\{\int _ { t _ { 0 } } ^ { t } \left(b_{2}(t)-a_{2}(t) x_{2}(t)-\frac{x_{2}(t)}{\mu_{2}(t)+\omega_{2}(t) x_{1}(t)}\right.\right. \\
y_{j}(t)=y_{j}\left(t_{k}^{+}\right) \exp \left\{\int _ { t _ { 0 } } ^ { t } \left(-\sum_{k=1}^{n} \frac{\left.\left.c_{2 k}(t) y_{k}(t)+\sum_{k=1}^{2} \frac{d_{j k}(t) x_{k}(t)}{\alpha_{j k}(t)+\beta_{j k}(t) x_{k}(t)+\gamma_{j k}(t) y_{j}(t)}\right) d t\right\},}{\left.\left.-\sum_{k=1}^{n} \delta_{j k}(t) y_{k}(t)\right) d t\right\},}\right.\right.
\end{gathered}
$$

for $t \in\left(t_{k}, t_{k+1}\right]$. So $x_{i}(t)>0, y_{j}(t)>0$ for $t \in J$. 


\section{Existence of Positive Periodic Solution}

We denote

$$
\begin{gathered}
\Delta_{i}=\bar{b}_{i}+\frac{1}{T} \sum_{k=1}^{q} \ln \left(1+b_{i k}\right), \quad \widetilde{\Delta}_{j}=\frac{1}{T} \sum_{k=1}^{q} \ln \left(1+\widetilde{b}_{j k}\right)-\bar{r}_{j}, \\
B=\left(\begin{array}{cc}
\bar{a}_{i^{*} j^{*}} & \mathbf{0} \\
\mathbf{0} & \bar{\delta}_{i^{\prime} j^{\prime}}
\end{array}\right), \quad \Delta=\left(\begin{array}{c}
\Delta_{i} \\
\tilde{\Delta}_{j}
\end{array}\right)
\end{gathered}
$$

where $i^{*}, j^{*} \in\{1, \ldots, n\}, i^{\prime}, j^{\prime} \in\{1, \ldots, n\}$. We also denote $B_{k}(k \in\{1, \ldots, n+2\})$ the matrix obtained by replacing the $k$ th column of $B$ with $\Delta$.

Theorem 3.1. If system (2.3) satisfies (A1)-(A5) and following conditions

(A6) $\Delta_{1}>\left(\left(\exp \left\{H_{1}\right\} / K_{2}\right)\left(\overline{1 / \omega_{1}}\right)-\sum_{k=1}^{n}\left(\overline{c_{1 k} / \gamma_{1 k}}\right)\right) / \bar{a}_{1}, \Delta_{2}>\left(\left(\exp \left\{H_{2}\right\} / K_{1}\right)\left(\overline{1 / \omega_{2}}\right)-\right.$ $\left.\sum_{k=1}^{n}\left(\overline{\left(c_{2 k} / \gamma_{2 k}\right.}\right)\right) / a_{2}, \widetilde{\Delta}_{j}>\sum_{k=1, k \neq j}^{n} \bar{\delta}_{j k} \exp \left\{\widetilde{H}_{k}\right\} / \bar{\delta}_{j j}$, where

$$
\begin{gathered}
H_{i}=\max \left\{\ln \left(\frac{\Delta_{i}}{\overline{\bar{a}}_{i i}}\right)\right\}+2 \sum_{k=1}^{q} \ln \left(1+b_{i k}\right), \\
\widetilde{H}_{j}=\max \left\{\ln \frac{\widetilde{\Delta}_{j}+\sum_{k=1}^{n}\left(\overline{d_{j k} / \beta_{j k}}\right)}{\bar{\delta}_{j j}}\right\}+2 \sum_{k=1}^{q} \ln \left(1+b_{j k}\right),
\end{gathered}
$$

(A7) $B>0, B_{k}>0, k \in\{1, \ldots, n+2\}$.

Then system (1.1) has at least one positive T-periodic solution.

Proof. Let $\mathbb{X}=P C_{T}^{1} \times P C_{T}^{1} \times \cdots \times P C_{T}^{1}$ be the Cartesian production of $(n+2)$-tuples, $\mathbb{Z}=$ $\mathbb{X} \times \mathbb{R}^{(n+2) q}$, where $\mathbb{R}^{(n+2) q}$ is $(n+2) q$-dimensional Euclidean space. It is clear that $\mathbf{x}(0)=\mathbf{x}(T)$ for any $\mathbf{x} \in \mathbb{X}$.

We define

$$
\begin{gathered}
\|\mathbf{x}\|=\sum_{i=1}^{2}\left\|x_{i}\right\|_{P C_{T}^{1}}+\sum_{j=1}^{n}\left\|y_{j}\right\|_{P C_{T}^{1}}=\sum_{i=1}^{2} \sup _{[0, T]}\left|x_{i}(t)\right|+\sum_{j=1}^{n} \sup _{[0, T]}\left|y_{j}(t)\right|, \\
\|\mathbf{z}\|_{\mathbb{Z}}=\|\mathbf{x}\|+\|\mathbf{c}\|_{*^{\prime}}
\end{gathered}
$$

for any $\mathbf{x}=\left(x_{1}, x_{2}, y_{1}, \ldots, y_{n}\right) \in \mathbb{X}, \mathbf{c}=\left(c_{1}, \ldots, c_{(n+2) q}\right) \in \mathbb{R}^{(n+2) q}$, and $\mathbf{z}=(\mathbf{x}, \mathbf{c}) \in \mathbb{Z}$, where $\|\cdot\|$ is the norm of $\mathbb{X}$, and $\|\cdot\|_{*}$ is any norm of $\mathbb{R}^{(n+2) q}$. Then it is trivial to check that $\mathbb{X}, \mathbb{Z}$ are both Banach spaces when they are endowed with the above norms $\|\cdot\|$ and $\|\cdot\|_{\mathbb{Z}}$, respectively. 
Let

$$
\begin{gathered}
\mathcal{L}: \operatorname{Dom} \mathcal{L} \subset \mathbb{X} \rightarrow \mathbb{Z},\left(\begin{array}{c}
u_{i}(t) \\
v_{j}(t)
\end{array}\right) \longrightarrow\left(\left(\begin{array}{c}
\dot{u}_{i}(t) \\
\dot{v}_{j}(t)
\end{array}\right),\left\{\left(\begin{array}{c}
\Delta u_{i}\left(t_{k}\right) \\
\Delta v_{j}\left(t_{k}\right)
\end{array}\right)\right\}_{k=1}^{q}\right) \\
\mathcal{N}: \mathbb{X} \rightarrow \mathbb{Z}, \mathcal{N}\left(\begin{array}{c}
u_{i}(t) \\
v_{j}(t)
\end{array}\right)=\left(\left(\begin{array}{c}
\dot{u}_{i}(t) \\
\dot{v}_{j}(t)
\end{array}\right),\left\{\left(\begin{array}{c}
\Delta u_{i}\left(t_{k}\right) \\
\Delta v_{j}\left(t_{k}\right)
\end{array}\right)\right\}_{k=1}^{q}\right) .
\end{gathered}
$$

Then

$$
\begin{gathered}
\operatorname{Ker} \mathcal{L}=\left\{\left(\begin{array}{l}
u_{i}(t) \\
v_{j}(t)
\end{array}\right):\left(\begin{array}{c}
u_{i}(t) \\
v_{j}(t)
\end{array}\right)=\left(\begin{array}{c}
c_{1} \\
\vdots \\
c_{n+2}
\end{array}\right) \in \mathbb{R}^{n+2}\right\}, \\
\operatorname{Im} \mathcal{L}=\left\{\mathbf{z}=\left(\left(\begin{array}{c}
f_{\mathrm{i}} \\
\tilde{f}_{j}
\end{array}\right),\left\{\left(\begin{array}{c}
l_{i k} \\
\tilde{l}_{j k}
\end{array}\right)\right\}_{k=1}^{q}\right) \in \mathbb{Z}: \int_{0}^{T} f_{i} d t+\sum_{k=1}^{q} l_{i k}=0, \int_{0}^{T} \tilde{f}_{j} d t+\sum_{k=1}^{q} \tilde{l}_{j k}=0\right\},
\end{gathered}
$$

$\operatorname{dim} \operatorname{Ker} \mathcal{L}=n+2=$ codim $\operatorname{Im} \mathcal{L}$.

Since $\operatorname{Im} \perp$ is closed in $\mathbb{Z}, \perp$ is a Fredholm mapping of index zero. We respectively define $P$ and $Q$ in the following:

$$
\begin{aligned}
& p\left(\begin{array}{c}
u_{i}(t) \\
v_{j}(t)
\end{array}\right)=\frac{1}{T}\left(\begin{array}{l}
\int_{0}^{T} u_{i}(t) d t \\
\int_{0}^{T} v_{j}(t) d t
\end{array}\right) \\
& \left.Q \mathrm{z}=Q\left(\left(\begin{array}{c}
f_{i} \\
\tilde{f}_{j}
\end{array}\right),\left\{\left(\begin{array}{c}
l_{i k} \\
\tilde{l}_{j k}
\end{array}\right)\right\}_{k=1}^{q}\right)=\left(\begin{array}{c}
\int_{0}^{T} f_{i} d t+\sum_{k=1}^{q} l_{i k} \\
\frac{1}{T}\left(\int_{0}^{T} \tilde{f}_{j} d t+\sum_{k=1}^{q} \tilde{l}_{j, k}\right.
\end{array}\right),\left\{\left(\begin{array}{c}
0 \\
\vdots \\
0
\end{array}\right)\right\}_{k=1}^{q}\right) .
\end{aligned}
$$

It is easy to show that $D, Q$ are continuous projectors such that $\operatorname{Im} \mathcal{L}=\operatorname{Ker} Q=\operatorname{Im}(I-Q)$. Furthermore, the generalized inverse $(\mathcal{L}) K_{p}: \operatorname{Im} \mathcal{L} \rightarrow \operatorname{Dom} \mathcal{L} \cap \operatorname{Ker} \perp$ exists. That follows one will calculate $K_{p}$.

Let

$$
\mathbf{z}=\left(\left(\begin{array}{c}
f_{i} \\
\tilde{f}_{j}
\end{array}\right),\left\{\left(\begin{array}{c}
l_{i k} \\
\tilde{l}_{j k}
\end{array}\right)\right\}_{k=1}^{q}\right) \in \operatorname{Im} \mathcal{L},
$$

then

$$
\left(\begin{array}{c}
\dot{u}_{i}(t) \\
\dot{v}_{j}(t)
\end{array}\right)=\left(\begin{array}{c}
f_{i}(t) \\
\tilde{f}_{j}(t)
\end{array}\right)_{t \neq t_{k}}, \quad\left(\begin{array}{c}
\Delta u_{i}(t) \\
\Delta v_{j}(t)
\end{array}\right)_{t=t_{k}}=\left(\begin{array}{c}
l_{i k} \\
\tilde{l}_{j k}
\end{array}\right)
$$


that is,

$$
\left(\begin{array}{l}
u_{i}(t) \\
v_{j}(t)
\end{array}\right)=\left(\begin{array}{l}
\int_{0}^{t} f_{i}(s) d s+\sum_{t>t_{k}} l_{i k}+u_{i}(0) \\
\int_{0}^{t} \tilde{f}_{j}(s) d s+\sum_{t>t_{k}} \tilde{l}_{j k}+v_{j}(0)
\end{array}\right)
$$

Note that

$$
\left(\begin{array}{l}
u_{i}(t) \\
v_{j}(t)
\end{array}\right) \in \operatorname{Ker} p, \text { that is, }\left(\begin{array}{l}
\frac{1}{T} \int_{0}^{T} u_{i}(s) d s \\
\frac{1}{T} \int_{0}^{T} v_{j}(s) d s
\end{array}\right)=\left(\begin{array}{l}
\mathbf{0} \\
\mathbf{0}
\end{array}\right)
$$

From(3.9), we obtain

$$
\left(\begin{array}{l}
\frac{1}{T} \int_{0}^{T} \int_{0}^{t} u_{i}(s) d s d t+\int_{0}^{T} \sum_{t>t_{k}} l_{i k} d t+T u_{i}(0) \\
\frac{1}{T} \int_{0}^{T} \int_{0}^{t} v_{j}(s) d s d t+\int_{0}^{T} \sum_{t>t_{k}} \tilde{l}_{j k} d t+T v_{j}(0)
\end{array}\right)=\left(\begin{array}{l}
\mathbf{0} \\
0
\end{array}\right)
$$

and hence,

$$
\left(\begin{array}{l}
u_{i}(t) \\
v_{j}(t)
\end{array}\right)=\left(\begin{array}{l}
\int_{0}^{t} u_{i}(s) d s+\sum_{t>t_{k}} l_{i k}-\frac{1}{T} \int_{0}^{T} \int_{0}^{t} u_{i}(s) d s d t-\sum_{k=1}^{q} l_{i k}+\frac{1}{T} \sum_{k=1}^{q} l_{i k} t_{k} \\
\int_{0}^{t} v_{j}(s) d s+\sum_{t>t_{k}} \widetilde{l}_{j k}-\frac{1}{T} \int_{0}^{T} \int_{0}^{t} v_{j}(s) d s d t-\sum_{k=1}^{q} \tilde{l}_{j k}+\frac{1}{T} \sum_{k=1}^{q} \tilde{l}_{j k} t_{k}
\end{array}\right)
$$

that is,

$$
K_{p} \mathrm{Z}=\left(\begin{array}{l}
\int_{0}^{t} f_{i}(s) d s+\sum_{t>t_{k}} l_{i k}-\frac{1}{T} \int_{0}^{T} \int_{0}^{t} f_{i}(s) d s d t-\sum_{k=1}^{q} l_{i k} \\
\int_{0}^{t} \tilde{f}_{j}(s) d s+\sum_{t>t_{k}} \tilde{l}_{j k}-\frac{1}{T} \int_{0}^{T} \int_{0}^{t} \tilde{f}_{j}(s) d s d t-\sum_{k=1}^{q} \tilde{l}_{j k}
\end{array}\right) .
$$

Thus

$$
Q \mathcal{N}=\left(\begin{array}{c}
u_{i}(t) \\
v_{j}(t)
\end{array}\right)=\left(\left(\begin{array}{l}
h_{i} \\
\widetilde{h}_{j}
\end{array}\right),\left\{\left(\begin{array}{l}
0 \\
0
\end{array}\right)\right\}_{k=1}^{q}\right),
$$


where

$$
\begin{gathered}
h_{1}=\Delta_{1}-\frac{1}{T} \int_{0}^{T}\left(a_{1}(t) \exp \left\{u_{1}(t)\right\}+\frac{\exp \left\{u_{1}(t)\right\}}{\mu_{1}(t)+\omega_{1}(t) \exp \left\{u_{2}(t)\right\}}\right. \\
\left.+\sum_{k=1}^{n} \frac{c_{1 k}(t) \exp \left\{v_{k}(t)\right\}}{\alpha_{1 k}(t)+\beta_{1 k}(t) \exp \left\{u_{1}(t)\right\}+\gamma_{1 k}(t) \exp \left\{v_{k}(t)\right\}}\right) d t \\
h_{2}=\Delta_{2}-\frac{1}{T} \int_{0}^{T}\left(a_{2}(t) \exp \left\{u_{2}(t)\right\}+\frac{\exp \left\{u_{2}(t)\right\}}{\mu_{1}(t)+\omega_{1}(t) \exp \left\{u_{1}(t)\right\}}\right. \\
\left.+\sum_{k=1}^{n} \frac{c_{2 k}(t) \exp \left\{v_{k}(t)\right\}}{\alpha_{2 k}(t)+\beta_{2 k}(t) \exp \left\{u_{2}(t)\right\}+\gamma_{2 k}(t) \exp \left\{v_{k}(t)\right\}}\right) d t, \\
\tilde{h}_{j}=\tilde{\Delta}_{j}+\frac{1}{T} \int_{0}^{T}\left(\sum_{k=1}^{2} \frac{d_{j k}(t) \exp \left\{u_{k}(t)\right\}}{\alpha_{j k}(t)+\beta_{j k}(t) \exp \left\{u_{k}(t)\right\}+\gamma_{j k}(t) \exp \left\{v_{j}(t)\right\}}\right. \\
\left.-\sum_{k=1}^{n} \delta_{j k}(t) \exp \left\{v_{k}(t)\right\}\right) d t .
\end{gathered}
$$

Clearly, $Q \mathcal{N}$ and $K_{p}(I-Q) \mathcal{N}$ are continuous. Using the Arzela-Ascoli theorem, it is not diffcult to show that $\overline{K_{p}(I-Q) \mathcal{\Omega}(\bar{\Omega})}$ is compact for any open bounded set $\Omega \subset \mathbb{X}$. Moreover, $Q \mathcal{N}(\Omega)$

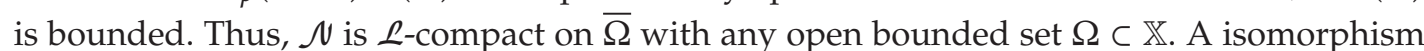
$\partial$ of $\operatorname{Im} Q$ onto $\operatorname{Ker} \mathcal{L}$ can be chosen to be the identity mapping; $\operatorname{since} \operatorname{Im} Q=\operatorname{Ker} \mathcal{L}$, there exists an isomorphism $\mathcal{2}: \operatorname{Im} Q \rightarrow \mathbb{X}$ given by

$$
\partial: \operatorname{Im} Q \longrightarrow \mathbb{X},\left(\left(\begin{array}{c}
g_{1} \\
\vdots \\
g_{n+2}
\end{array}\right),\left\{\left(\begin{array}{c}
0 \\
\vdots \\
0
\end{array}\right)\right\}_{k=1}^{q}\right) \longrightarrow\left(\begin{array}{c}
g_{1} \\
\vdots \\
g_{n+2}
\end{array}\right)
$$

Corresponding to operator equation $\mathcal{L} \mathbf{u}=\lambda \mathcal{N} \mathbf{u}, \mathbf{u}(t)=\left(u_{1}(t), u_{2}(t), v_{1}(t), \ldots, v_{n}(t)\right)^{T}, \lambda \in$ $(0,1)$, we have

$$
\begin{array}{r}
\dot{u}_{1}(t)=\lambda\left(b_{1}(t)-a_{1}(t) \exp \left\{u_{1}(t)\right\}-\frac{\exp \left\{u_{1}(t)\right\}}{\mu_{1}(t)+\omega_{1}(t) \exp \left\{u_{2}(t)\right\}}\right. \\
\left.\quad-\sum_{k=1}^{n} \frac{c_{1 k}(t) \exp \left\{v_{k}(t)\right\}}{\alpha_{1 k}(t)+\beta_{1 k}(t) \exp \left\{u_{1}(t)\right\}+\gamma_{1 k}(t) \exp \left\{v_{k}(t)\right\}}\right),
\end{array}
$$




$$
\begin{gathered}
\dot{u}_{2}(t)=\lambda\left(b_{2}(t)-a_{2}(t) \exp \left\{u_{2}(t)\right\}-\frac{\exp \left\{u_{2}(t)\right\}}{\mu_{1}(t)+\omega_{1}(t) \exp \left\{u_{1}(t)\right\}}\right. \\
\left.-\sum_{k=1}^{n} \frac{c_{2 k}(t) \exp \left\{v_{k}(t)\right\}}{\alpha_{2 k}(t)+\beta_{2 k}(t) \exp \left\{u_{2}(t)\right\}+\gamma_{2 k}(t) \exp \left\{v_{k}(t)\right\}}\right), \\
\dot{v}_{j}(t)=\lambda\left(-r_{j}(t)+\sum_{k=1}^{2} \frac{d_{j k}(t) \exp \left\{u_{k}(t)\right\}}{\alpha_{j k}(t)+\beta_{j k}(t) \exp \left\{u_{k}(t)\right\}+\gamma_{j k}(t) \exp \left\{v_{j}(t)\right\}}\right. \\
\left.-\sum_{k=1}^{n} \delta_{j k}(t) \exp \left\{v_{k}(t)\right\}\right), \\
\Delta u_{i}(t)=u_{i}\left(t^{+}\right)-u_{i}\left(t^{-}\right)=\lambda \ln \left(1+b_{i k}\right), \\
\Delta v_{j}(t)=v_{j}\left(t^{+}\right)-v_{j}\left(t^{-}\right)=\lambda \ln \left(1+\tilde{b}_{j k}\right), \\
t=t_{k}, \quad\left(k \in N^{+}\right) .
\end{gathered}
$$

Suppose that $\mathbf{u}(t)=\left(u_{1}(t), u_{2}(t), v_{1}(t), \ldots, v_{n}(t)\right)^{T}$ is a solution of system (3.17) for a fixed $\lambda \in(0,1)$. Integrating on both sides of (3.17) from 0 to $T$, we obtain

$$
\begin{aligned}
& \int_{0}^{T}\left(-b_{1}(t)+a_{1}(t) \exp \left\{u_{1}(t)\right\}+\frac{\exp \left\{u_{1}(t)\right\}}{\mu_{1}(t)+\omega_{1}(t) \exp \left\{u_{2}(t)\right\}}\right. \\
& \left.\quad+\sum_{k=1}^{n} \frac{c_{1 k}(t) \exp \left\{v_{k}(t)\right\}}{\alpha_{1 k}(t)+\beta_{1 k}(t) \exp \left\{u_{1}(t)\right\}+\gamma_{1 k}(t) \exp \left\{v_{k}(t)\right\}}\right) d t \\
& \quad=\sum_{k=1}^{q} \ln \left(1+b_{1 k}\right), \\
& \int_{0}^{T}\left(-b_{2}(t)+a_{2}(t) \exp \left\{u_{2}(t)\right\}+\frac{\exp \left\{u_{2}(t)\right\}}{\mu_{2}(t)+\omega_{2}(t) \exp \left\{u_{1}(t)\right\}}\right. \\
& \left.\quad+\sum_{k=1}^{n} \frac{c_{2 k}(t) \exp \left\{v_{k}(t)\right\}}{\alpha_{2 k}(t)+\beta_{2 k}(t) \exp \left\{u_{2}(t)\right\}+\gamma_{2 k}(t) \exp \left\{v_{k}(t)\right\}}\right) d t \\
& =\sum_{k=1}^{q} \ln \left(1+b_{2 k}\right),
\end{aligned}
$$




$$
\begin{gathered}
\int_{0}^{T}\left(r_{j}(t)-\sum_{k=1}^{2} \frac{d_{j k}(t) \exp \left\{u_{k}(t)\right\}}{\alpha_{j k}(t)+\beta_{j k}(t) \exp \left\{u_{k}(t)\right\}+\gamma_{j k}(t) \exp \left\{v_{j}(t)\right\}}\right. \\
\left.+\sum_{k=1}^{n} \delta_{j k}(t) \exp \left\{v_{k}(t)\right\}\right) d t=\sum_{k=1}^{q} \ln \left(1+\tilde{b}_{j k}\right) .
\end{gathered}
$$

Since $\mathbf{u} \in \mathbb{X}$, there exist $\xi_{i}, \xi_{j} \in[0, T]$ such that

$$
u_{i}\left(\xi_{i}\right)=\min _{t \in[0, T]} u_{i}(t), \quad v_{j}\left(\xi_{j}\right)=\min _{t \in[0, T]} v_{j}(t)
$$

On the other hand, note that there exist $\zeta_{i}^{+}, \zeta_{j}^{+} \in[0, T]$ such that

$$
u_{i}\left(\zeta_{i}^{+}\right)=\sup _{t \in[0, T]} u_{i}(t), \quad v_{j}\left(\zeta_{j}^{+}\right)=\sup _{t \in[0, T]} v_{j}(t)
$$

If $\zeta_{i} \neq t_{k}$, then $u_{i}\left(\zeta_{i}^{+}\right)=u_{i}\left(\zeta_{i}\right), v_{j}\left(\zeta_{j}^{+}\right)=v_{j}\left(\zeta_{j}\right)$. While if $\zeta_{j}=t_{k}$, we have $u_{i}\left(\zeta_{i}^{+}\right)=u_{i}\left(t_{k}^{+}\right), v_{j}\left(\zeta_{j}^{+}\right)=$ $v_{j}\left(t_{k}^{+}\right)$. Thus we can obtain from (3.18) and (3.19) that

$$
\begin{aligned}
\int_{0}^{T} a_{i}(t) \exp \left\{u_{i}\left(\xi_{i}\right)\right\} d t & \leqslant \int_{0}^{T} a_{i}(t) e^{u_{i}(t)} d t \\
& \leqslant \sum_{k=1}^{q} \ln \left(1+b_{i k}\right)+\int_{0}^{T} b_{i}(t) d t \\
\int_{0}^{T} \delta_{j j}(t) \exp \left\{v_{j}\left(\xi_{j}\right)\right\} d t & \leqslant \int_{0}^{T} \delta_{j j}(t) \exp \left\{v_{j}(t)\right\} d t \\
& \leqslant \sum_{k=1}^{q} \ln \left(1+\tilde{b}_{j k}\right)-\int_{0}^{T} r_{j}(t) d t+\sum_{k=1}^{n} \int_{0}^{T} \frac{d_{j k}(t)}{\beta_{j k}(t)} d t .
\end{aligned}
$$

It follows that

$$
u_{i}\left(\xi_{i}\right) \leqslant \max \left\{\ln \frac{\Delta_{i}}{\bar{a}_{i}}\right\}:=p, \quad v_{j}\left(\xi_{j}\right) \leqslant \max \left\{\ln \frac{\tilde{\Delta}_{j}+\sum_{k=1}^{n}\left(\overline{d_{j k} / \beta_{j k}}\right)}{\bar{\delta}_{j j}}\right\}:=g .
$$

From (3.17) and (3.18), we obtain

$$
\int_{0}^{T}\left|\dot{u}_{i}(t)\right| d t \leqslant 2 \sum_{k=1}^{q} \ln \left(1+b_{i k}\right), \quad \int_{0}^{T}\left|\dot{v}_{j}(t)\right| d t \leqslant 2 \sum_{k=1}^{q} \ln \left(1+\tilde{b}_{j k}\right) .
$$


By (3.22) and (3.23), we have that

$$
\begin{gathered}
u_{i}(t) \leqslant u_{i}\left(\xi_{i}\right)+\int_{0}^{T}\left|\dot{u}_{i}(t)\right| d t \leqslant p+2 \sum_{k=1}^{q} \ln \left(1+b_{i k}\right):=H_{i}, \\
v_{j}(t) \leqslant v_{j}\left(\xi_{j}\right)+\int_{0}^{T}\left|\dot{v}_{j}(t)\right| d t \leqslant g+2 \sum_{k=1}^{q} \ln \left(1+\widetilde{b}_{j k}\right):=\widetilde{H}_{j} .
\end{gathered}
$$

On the other hand, by (3.18) and (3.20), we also have

$$
\begin{aligned}
\int_{0}^{T} \delta_{j j}(t) \exp \left\{v_{j}\left(\zeta_{j}^{+}\right)\right\} d t & \geqslant \int_{0}^{T} \delta_{j j}(t) \exp \left\{v_{j}(t)\right\} d t \\
& \geqslant \sum_{k=1}^{q} \ln \left(1+\widetilde{b}_{j k}\right)-\int_{0}^{T} r_{j}(t) d t-\sum_{k=1, k \neq j}^{n} \int_{0}^{T} \delta_{j k}(t) \exp \left\{\widetilde{H}_{k}\right\} d t \\
v_{j}\left(\zeta_{j}^{+}\right) & \geqslant \ln \left(\frac{\widetilde{\Delta}_{j}-\sum_{k=1, k \neq j}^{n} \bar{\delta}_{j k} \exp \left\{\widetilde{H}_{k}\right\}}{\bar{\delta}_{j j}}\right),
\end{aligned}
$$

and hence,

$$
\begin{aligned}
v_{j}(t) & \geqslant v_{j}\left(\zeta_{j}^{+}\right)-\int_{0}^{T}\left|\dot{v}_{j}(t)\right| d t \\
& \geqslant \ln \left(\frac{\widetilde{\Delta}_{j}-\sum_{k=1, k \neq j}^{n} \bar{\delta}_{j k} \exp \left\{\widetilde{H}_{k}\right\}}{\bar{\delta}_{j j}}\right)-2 \sum_{k=1}^{q} \ln \left(1+\widetilde{b}_{j k}\right):=\widetilde{M}_{j} .
\end{aligned}
$$

By (3.18), we have

$$
\begin{aligned}
\int_{0}^{T} a_{1}(t) \exp \left\{u_{1}\left(\zeta_{1}^{+}\right)\right\} d t \geqslant & \int_{0}^{T} a_{1}(t) \exp \left\{u_{1}(t)\right\} d t \\
\geqslant & \sum_{k=1}^{q} \ln \left(1+b_{1 k}\right)+\int_{0}^{T} b_{1}(t) d t-\int_{0}^{T} \frac{\exp \left\{u_{1}(t)\right\}}{\mu_{1}(t)+\omega_{1}(t) \exp \left\{u_{2}(t)\right\}} d t \\
& -\sum_{k=1}^{n} \int_{0}^{T} \frac{c_{1 k}(t) \exp \left\{v_{k}(t)\right\}}{\alpha_{1 k}(t)+\beta_{1 k}(t) \exp \left\{u_{1}(t)\right\}+\gamma_{1 k}(t) \exp \left\{v_{k}(t)\right\}} d t \\
\geqslant & \sum_{k=1}^{q} \ln \left(1+b_{1 k}\right)+\int_{0}^{T} b_{1}(t) d t-\frac{\exp \left\{H_{1}\right\}}{K_{2}} \int_{0}^{T} \frac{1}{\omega_{1}(t)} d t \\
& -\sum_{k=1}^{n} \int_{0}^{T} \frac{c_{1 k}(t)}{\gamma_{1 k}(t)} d t
\end{aligned}
$$




$$
\begin{aligned}
\int_{0}^{T} a_{2}(t) \exp \left\{u_{2}\left(\zeta_{2}^{+}\right)\right\} d t \geqslant & \int_{0}^{T} a_{2}(t) \exp \left\{u_{2}(t)\right\} d t \\
\geqslant & \sum_{k=1}^{q} \ln \left(1+b_{2 k}\right)+\int_{0}^{T} b_{2}(t) d t-\int_{0}^{T} \frac{\exp \left\{u_{2}(t)\right\}}{\mu_{2}(t)+\omega_{2}(t) \exp \left\{u_{1}(t)\right\}} d t \\
& -\sum_{k=1}^{n} \int_{0}^{T} \frac{c_{2 k}(t) \exp \left\{v_{k}(t)\right\}}{\alpha_{2 k}(t)+\beta_{1 k}(t) \exp \left\{u_{2}(t)\right\}+\gamma_{2 k}(t) \exp \left\{v_{k}(t)\right\}} d t \\
\geqslant & \sum_{k=1}^{q} \ln \left(1+b_{2 k}\right)+\int_{0}^{T} b_{2}(t) d t-\frac{\exp \left\{H_{2}\right\}}{K_{1}} \int_{0}^{T} \frac{1}{\omega_{2}(t)} d t \\
& -\sum_{k=1}^{n} \int_{0}^{T} \frac{c_{2 k}(t)}{\gamma_{2 k}(t)} d t
\end{aligned}
$$

where $K_{i}$ is a positive number satisfied $\exp \left\{u_{i}(t)\right\} \geqslant K_{i}$ for $t \in[0, T]$; therefore,

$$
\begin{aligned}
& u_{1}\left(\zeta_{1}^{+}\right) \geqslant \ln \left(\frac{\Delta_{1}-\left(\exp \left\{H_{1}\right\} / K_{2}\right)\left(\overline{1 / \omega_{1}}\right)-\sum_{k=1}^{n}\left(\overline{c_{1 k} / \gamma_{1 k}}\right)}{\bar{a}_{1}}\right), \\
& u_{2}\left(\zeta_{2}^{+}\right) \geqslant \ln \left(\frac{\Delta_{2}-\left(\exp \left\{H_{2}\right\} / K_{1}\right)\left(\overline{1 / \omega_{2}}\right)-\sum_{k=1}^{n}\left(\overline{c_{2 k} / \gamma_{2 k}}\right)}{\bar{a}_{2}}\right) .
\end{aligned}
$$

So

$$
\begin{aligned}
u_{1}(t) & \geqslant u_{1}\left(\zeta_{1}^{+}\right)-\int_{0}^{T}\left|\dot{u}_{1}(t)\right| d t \\
& \geqslant \ln \left(\frac{\Delta_{1}-\left(\exp \left\{H_{1}\right\} / K_{2}\right)\left(\overline{1 / \omega_{1}}\right)-\sum_{k=1}^{n}\left(\overline{c_{1 k} / \gamma_{1 k}}\right)}{\bar{a}_{1}}\right)-2 \sum_{k=1}^{q} \ln \left(1+b_{1 k}\right):=N_{1}, \\
u_{2}(t) & \geqslant u_{2}\left(\zeta_{2}^{+}\right)-\int_{0}^{T}\left|\dot{u}_{2}(t)\right| d t \\
& \geqslant \ln \left(\frac{\Delta_{2}-\left(\exp \left\{H_{2}\right\} / K_{1}\right)\left(\overline{1 / \omega_{2}}\right)-\sum_{k=1}^{n}\left(\overline{c_{2 k} / \gamma_{2 k}}\right)}{\bar{a}_{2}}\right)-2 \sum_{k=1}^{q} \ln \left(1+b_{2 k}\right):=N_{2} .
\end{aligned}
$$


Take $M_{i}=\min \left\{K_{i}, N_{i}\right\}$. By (3.24)-(3.29), we have

$$
\left|u_{i}(t)\right|<\max \left\{\left|M_{i}\right|,\left|H_{i}\right|\right\}:=G_{i}, \quad\left|v_{j}(t)\right|<\max \left\{\left|\widetilde{M}_{j}\right|,\left|\widetilde{H}_{j}\right|\right\}:=\widetilde{G}_{j} .
$$

Here, $G_{i}, \widetilde{G}_{j}$ are independent of the choice of $\lambda$.

Let $\mathbf{u}=\left(u_{1}(t), u_{2}(t), v_{1}(t), \ldots, v_{n}(t)\right)^{T} \in \mathbb{R}^{n+2}$, set $G=\|\mathbf{u}\|+G_{0}$, where $G_{0}$ is taken sufficiently large, such that the solution $\mathbf{u}^{*}=\left(u_{1}^{*}, u_{2}^{*}, v_{1}^{*}, \ldots, v_{n}^{*}\right)^{T}$ of the equation

$$
\begin{aligned}
\bar{b}_{1}-\bar{a}_{1} \exp \left\{u_{1}(t)\right\}-\frac{1}{T} \int_{0}^{T} \frac{\exp \left\{u_{1}(t)\right\} d t}{\mu_{1}(t)+\omega_{1}(t) \exp \left\{u_{2}(t)\right\}} \\
-\frac{1}{T} \sum_{k=1}^{n} \int_{0}^{T} \frac{c_{1 k}(t) \exp \left\{v_{k}(t)\right\} d t}{\alpha_{1 k}(t)+\beta_{1 k}(t) \exp \left\{u_{1}(t)\right\}+\gamma_{1 k}(t) \exp \left\{v_{k}(t)\right\}} \\
+\frac{1}{T} \sum_{k=1}^{q} \ln \left(1+b_{1 k}\right)=0, \\
\bar{b}_{2}-\bar{a}_{2} \exp \left\{u_{2}(t)\right\}-\frac{1}{T} \int_{0}^{T} \frac{\exp \left\{u_{2}(t)\right\} d t}{\mu_{2}(t)+\omega_{2}(t) \exp \left\{u_{1}(t)\right\}} \\
-\frac{1}{T} \sum_{k=1}^{n} \int_{0}^{T} \frac{c_{2 k}(t) \exp \left\{v_{k}(t)\right\} d t}{\alpha_{2 k}(t)+\beta_{2 k}(t) \exp \left\{u_{2}(t)\right\}+\gamma_{2 k}(t) \exp \left\{v_{k}(t)\right\}} \\
+\frac{1}{T} \sum_{k=1}^{q} \ln \left(1+b_{2 k}\right)=0, \\
-\bar{r}_{j}+\frac{1}{T} \sum_{k=1}^{2} \int_{0}^{T} \frac{d_{j k}(t) \exp \left\{u_{k}(t)\right\} d t}{\alpha_{j k}(t)+\beta_{j k}(t) \exp \left\{u_{k}(t)\right\}+\gamma_{j k}(t) \exp \left\{v_{j}(t)\right\}} \\
\quad-\sum_{k=1}^{n} \bar{\delta}_{j k} \exp \left\{v_{k}(t)\right\}+\frac{1}{T} \sum_{k=1}^{q} \ln \left(1+\tilde{b}_{j k}\right)=0
\end{aligned}
$$

satisfies

$$
\begin{gathered}
\left\|\left(u_{1}^{*}, u_{2}^{*}, v_{1}^{*}, \ldots, v_{n}^{*}\right)^{T}\right\|<G_{0}, \\
\left\|\mathbf{u}^{*}\left(t_{k}+0\right)\right\|<G, \quad(k=1,2, \ldots, q) .
\end{gathered}
$$

Let $\Omega=\left\{\mathbf{u}=\left(u_{1}, u_{2}, v_{1}, \ldots, v_{n}\right)^{T} \in \mathbb{X} \mid\left\|\left(u_{1}, u_{2}, v_{1}, \ldots, v_{n}\right)^{T}\right\|<G\right\}$. Then it is clear that $\Omega$ satisfies the requirement (a) of Lemma 2.3. 
When $\mathbf{u} \in \partial \Omega \cap \operatorname{Ker} \mathcal{L}=\partial \Omega \cap \mathbb{R}^{n+2}, \mathbf{u}$ is a constant vector in $\mathbb{R}^{n+2}$ with $\|\mathbf{u}\|=G$, then $Q \mathcal{N} \neq 0$, we check $Q \mathcal{N} \mathbf{u}$ and according to mean value theorem, there exist $\theta_{1}, \theta_{2}, \tilde{\theta}_{j}, \eta_{1}, \eta_{2} \in$ $[0, T]$ such that

$$
\left.Q \boldsymbol{N u}=\left(\begin{array}{c}
\Delta_{1}-\bar{a}_{1} \exp \left\{u_{1}(t)\right\}-\frac{\exp \left\{u_{1}(t)\right\}}{\mu_{1}\left(\theta_{1}\right)+\omega_{1}\left(\theta_{1}\right) \exp \left\{u_{2}(t)\right\}} \\
-\sum_{k=1}^{n} \frac{\bar{c}_{1 k} \exp \left\{v_{k}(t)\right\}}{\alpha_{1 k}\left(\eta_{1}\right)+\beta_{1 k}\left(\eta_{1}\right) \exp \left\{u_{1}(t)\right\}+\gamma_{1 k}\left(\eta_{1}\right) \exp \left\{v_{k}(t)\right\}} \\
\Delta_{2}-\bar{a}_{2} \exp \left\{u_{2}(t)\right\}-\frac{\exp \left\{u_{2}(t)\right\}}{\mu_{2}\left(\theta_{2}\right)+\omega_{2}\left(\theta_{2}\right) \exp \left\{u_{1}(t)\right\}} \\
-\sum_{k=1}^{n} \frac{\bar{c}_{2 k} \exp \left\{v_{k}(t)\right\}}{\alpha_{2 k}\left(\eta_{2}\right)+\beta_{2 k}\left(\eta_{2}\right) \exp \left\{u_{2}(t)\right\}+\gamma_{2 k}\left(\eta_{2}\right) \exp \left\{v_{k}(t)\right\}} \\
\tilde{\Delta}_{j}+\sum_{k=1}^{2} \frac{\exp \left\{u_{k}(t)\right\}}{\alpha_{j k}\left(\tilde{\theta}_{j}\right)+\beta_{j k}\left(\tilde{\theta}_{j}\right) \exp \left\{u_{k}(t)\right\}+\gamma_{j k}\left(\tilde{\theta}_{j}\right) \exp \left\{v_{j}(t)\right\}} \\
-\sum_{k=1}^{n} \bar{\delta}_{j k} \exp \left\{v_{k}(t)\right\}
\end{array}\right)\left(\begin{array}{c}
0 \\
\vdots \\
0
\end{array}\right)\right\}_{k=1}^{q}
$$

For any $\mathbf{u} \in \Omega \cap \operatorname{Ker} \mathcal{L}$, we have

$$
2 Q \boldsymbol{N} \mathbf{u}=\left(\begin{array}{c}
\Delta_{1}-\bar{a}_{1} \exp \left\{u_{1}(t)\right\}-\frac{\exp \left\{u_{1}(t)\right\}}{\mu_{1}\left(\theta_{1}\right)+\omega_{1}\left(\theta_{1}\right) \exp \left\{u_{2}(t)\right\}} \\
-\sum_{k=1}^{n} \frac{\bar{c}_{1 k} \exp \left\{v_{k}(t)\right\}}{\alpha_{1 k}\left(\eta_{1}\right)+\beta_{1 k}\left(\eta_{1}\right) \exp \left\{u_{1}(t)\right\}+\gamma_{1 k}\left(\eta_{1}\right) \exp \left\{v_{k}(t)\right\}} \\
\Delta_{2}-\bar{a}_{2} \exp \left\{u_{2}(t)\right\}-\frac{\exp \left\{u_{2}(t)\right\}}{\mu_{2}\left(\theta_{2}\right)+\omega_{2}\left(\theta_{2}\right) \exp \left\{u_{1}(t)\right\}} \\
-\sum_{k=1}^{n} \frac{\bar{c}_{2 k} \exp \left\{v_{k}(t)\right\}}{\alpha_{2 k}\left(\eta_{2}\right)+\beta_{2 k}\left(\eta_{2}\right) \exp \left\{u_{2}(t)\right\}+\gamma_{2 k}\left(\eta_{2}\right) \exp \left\{v_{k}(t)\right\}} \\
\tilde{\Delta}_{j k}+\sum_{k=1}^{2} \frac{\bar{d}_{j k} \exp \left\{u_{k}(t)\right\}}{\alpha_{j k}\left(\tilde{\theta}_{j}\right)+\beta_{j k}\left(\tilde{\theta}_{j}\right) \exp \left\{u_{k}(t)\right\}+\gamma_{j k}\left(\tilde{\theta}_{j}\right) \exp \left\{v_{j}(t)\right\}} \\
-\sum_{k=1}^{n} \bar{\delta}_{j k} \exp \left\{v_{k}(t)\right\}
\end{array}\right) .
$$

Define the map $\psi:\left(\bar{\Omega} \cap \mathbb{R}^{n+2}\right) \times[0,1] \rightarrow \mathbb{R}^{n+2}$ by

$$
\psi\left(\begin{array}{c}
u_{1} \\
u_{2} \\
v_{j}
\end{array}\right)=\left(\begin{array}{c}
\Delta_{1}-\bar{a}_{1} \exp \left\{u_{1}(t)\right\} \\
\Delta_{2}-\bar{a}_{2} \exp \left\{u_{2}(t)\right\} \\
\tilde{\Delta}_{j}-\sum_{k=1}^{n} \bar{\delta}_{j k} \exp \left\{v_{k}(t)\right\}
\end{array}\right)
$$




$$
-\mu\left(\begin{array}{l}
\frac{\exp \left\{u_{1}(t)\right\}}{\mu_{1}\left(\theta_{1}\right)+\omega_{1}\left(\theta_{1}\right) \exp \left\{u_{2}(t)\right\}} \\
+\sum_{k=1}^{n} \frac{\bar{c}_{1 k} \exp \left\{v_{k}(t)\right\}}{\alpha_{1 k}\left(\eta_{1}\right)+\beta_{1 k}\left(\eta_{1}\right) \exp \left\{u_{1}(t)\right\}+\gamma_{1 k}\left(\eta_{1}\right) \exp \left\{v_{k}(t)\right\}} \\
\frac{\exp \left\{u_{2}(t)\right\}}{\mu_{2}\left(\theta_{2}\right)+\omega_{2}\left(\theta_{2}\right) \exp \left\{u_{1}(t)\right\}} \\
+\sum_{k=1}^{n} \frac{\bar{c}_{2 k} \exp \left\{v_{k}(t)\right\}}{\alpha_{2 k}\left(\eta_{2}\right)+\beta_{2 k}\left(\eta_{2}\right) \exp \left\{u_{2}(t)\right\}+\gamma_{2 k}\left(\eta_{2}\right) \exp \left\{v_{k}(t)\right\}} \\
-\sum_{k=1}^{2} \frac{\bar{d}_{j k} \exp \left\{u_{k}(t)\right\}}{\alpha_{j k}\left(\tilde{\theta}_{j}\right)+\beta_{j k}\left(\tilde{\theta}_{j}\right) \exp \left\{u_{k}(t)\right\}+\gamma_{j k}\left(\tilde{\theta}_{j}\right) \exp \left\{v_{j}(t)\right\}}
\end{array}\right),
$$

where $\left(u_{1}, u_{2}, v_{1}, \ldots, v_{n}\right) \in \mathbb{R}^{n+2}, \mu \in[0,1]$. We can show that

$$
\psi\left(u_{1}, u_{2}, v_{1}, \ldots, v_{n}, \mu\right) \neq 0
$$

for any $\mathbf{u}=\left(u_{1}, u_{2}, v_{1}, \ldots, v_{n}\right)^{T} \in \partial \Omega \cap \mathbb{R}^{n+2}, \mu \in[0,1]$. Otherwise, it is similar to above mentioned discussion, we obtain that the solution $\mathbf{u}=\left(u_{1}, u_{2}, v_{1}, \ldots, v_{n}\right)^{T}$ of $\psi\left(u_{1}, u_{2}, v_{1}, \ldots, v_{n}, \mu\right)=0$ satisfies $\|\mathbf{u}\|<G$; therefore, this contradicts the fact that $\mathbf{u} \in$ $\partial \Omega \cap \mathbb{R}^{n+2}$.

Since $\psi\left(u_{1}, u_{2}, v_{1}, \ldots, v_{n}, \mu\right)$ is a homotopic mapping and topological degree is invariant under homotopic mapping, thus we can show the topological degree as folows:

$$
\begin{aligned}
\operatorname{deg}\left(\left(2 \Omega \mathcal{N}, \Omega \cap \operatorname{Ker} \mathcal{L},(0, \ldots, 0)^{T}\right)\right) & =\operatorname{deg}\left(\left(\psi_{\mu=1}, \Omega \cap \operatorname{Ker} \mathcal{L},(0, \ldots, 0)^{T}\right)\right) \\
& =\operatorname{deg}\left(\left(\psi_{\mu=0}, \Omega \cap \operatorname{Ker} \mathcal{L},(0, \ldots, 0)^{T}\right)\right) .
\end{aligned}
$$

On the other hand, the differential equations

$$
\begin{gathered}
\bar{b}_{i}-\bar{a}_{i} x_{k}^{*}+\frac{1}{T} \sum_{k=1}^{q} \ln \left(1+b_{i k}\right)=0, \\
-\bar{r}_{j}-\sum_{k=1}^{n} \bar{\delta}_{j k} y_{k}^{*}+\frac{1}{T} \sum_{k=1}^{q} \ln \left(1+\tilde{b}_{j k}\right)=0
\end{gathered}
$$


satisfies condition (A7), by the law of Crammer, one can easily obtain that (3.38) has unique solution

$$
\left(\widetilde{x_{1}^{*}}, \widetilde{x_{2}^{*}}, \widetilde{y_{1}^{*}}, \ldots, \widetilde{y_{n}^{*}}\right)
$$

where $\widetilde{x_{i}^{*}}=\operatorname{det}\left(B_{i}\right) / \operatorname{det}(B), \widetilde{y_{j}^{*}}=\operatorname{det}\left(B_{i}\right) / \operatorname{det}(B)$. Therefore, one can check

$$
\begin{aligned}
\operatorname{deg}\left(2 Q \mathcal{N}, \Omega \cap \operatorname{Ker} \mathcal{L},(0, \ldots, 0)^{T}\right) & =\operatorname{deg}\left(\psi_{\mu=0}, \Omega \cap \operatorname{Ker} \mathcal{L},(0, \ldots, 0)^{T}\right) \\
& =\operatorname{sgn}\left((-1)^{n+2} x_{1}^{*} x_{2}^{*} \prod_{j=1}^{n} y_{j}^{*} \operatorname{det}(B)\right) \neq 0 .
\end{aligned}
$$

By now we have proved that $\Omega$ satisfies all requirements of Lemma 2.3, then $\mathcal{L} \mathbf{x}=\mathcal{N} \mathbf{x}$

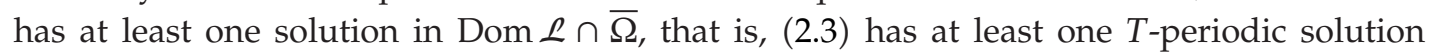
in Dom $\mathcal{L} \cap \bar{\Omega}$ and denote $\widehat{\mathbf{u}}=\left(\widehat{u}_{1}, \widehat{u}_{2}, \widehat{v}_{1}, \ldots, \widehat{v}_{n}\right)^{T}$ by the solution of system (2.3). Set $\widehat{x}_{i}=\exp \left\{\widehat{u}_{i}\right\}, \widehat{y}_{j}=\exp \left\{\widehat{v}_{j}\right\}$, then $\widehat{\mathbf{x}}=\left(\widehat{x}_{1}, \widehat{x}_{2}, \widehat{y}_{1}, \ldots, \widehat{y}_{n}\right)^{T}$ is one positive $T$-periodic solution of system (1.3). The proof is complete.

\section{Global Stability and Globally Exponential Stability of Solutions}

Let $\mathbf{x}_{0}=\left(x_{10}, x_{20}, y_{1,0}, \ldots, y_{n, 0}\right)$ and $x_{i 0}, y_{j 0} \in R_{+}$. We denote by

$$
\mathbf{x}(t)=\mathbf{x}\left(t ; t_{0}, x_{0}\right)=\left(x_{1}(t), x_{2}(t), y_{1}(t), \ldots, y_{n}(t)\right)
$$

the solution of system (1.3) satisfying the initial conditions:

$$
\mathbf{x}\left(t_{0}+0 ; t_{0}, x_{0}\right)=\mathbf{x}_{0}
$$

and by $J=J\left(t_{0}, \mathbf{x}_{0}\right)$ the maximal interval of type $\left[t_{0}, \eta\right)$ in which the solution $\mathbf{x}\left(t ; t_{0}, x_{0}\right)$ is defined.

Let $\mathbf{x}\left(t ; t_{0}, x_{0}\right), \mathbf{x}(t)=\left(x_{1}(t), x_{2}(t), y_{1}(t), \ldots, y_{n}(t)\right)$ and $\mathbf{x}^{*}\left(t ; t_{0}, x_{0}^{*}\right), \mathbf{x}^{*}(t)=\left(x_{1}^{*}(t), x_{2}^{*}(t)\right.$, $\left.y_{1}^{*}(t), \ldots, y_{n}^{*}(t)\right)$ be any two solutions of (1.1) with initial conditions:

$$
\mathbf{x}\left(t_{0}+0 ; t_{0}, x_{0}\right)=\mathbf{x}_{0}, \quad \mathbf{x}^{*}\left(t_{0}+0 ; t_{0}, x_{0}^{*}\right)=\mathbf{x}_{0}^{*}, \quad t_{0} \in R_{+} .
$$

We introduce the following notations:

$$
G_{k}=\left(t_{k-1}, t_{k}\right) \times \mathbb{R}_{+}^{n+2}, \quad k \in \mathbb{Z}^{+}, \quad G=\bigcup_{k=1}^{\infty} G_{k} .
$$

We put forward two definitions in [24, 28]. 
Definition 4.1 (Ahmad and Stamova [24]). The system (1.3) is said to be

(a) globally stable if for all $\varepsilon>0$, there exists $\delta=\delta\left(\varepsilon, t_{0}\right)>0$ such that if $\mathbf{x}(t), \mathbf{x}^{*}(t) \in \mathbb{R}_{+}^{n+2}$, with $\left\|\mathbf{x}_{0}-\mathbf{x}_{0}^{*}\right\| \leqslant \delta$ then for all $t \geqslant t_{0}\left\|\mathbf{x}\left(t ; t_{0}, x_{0}\right)-\mathbf{x}^{*}\left(t ; t_{0}, x_{0}^{*}\right)\right\|<\varepsilon$;

(b) globally asymptotically stable if it is globally stable and

$$
\lim _{t \rightarrow \infty}\left\|\mathbf{x}\left(t ; t_{0}, x_{0}\right)-\mathbf{x}^{*}\left(t ; t_{0}, x_{0}^{*}\right)\right\|=0
$$

(c) globally exponentially stable if for all $\alpha>0$, there exists $\gamma=\gamma(\alpha)>0$ such that $\mathbf{x}(t), \mathbf{x}^{*}(t) \in \mathbb{R}_{+}^{n+2}$, with $\left\|\mathbf{x}_{0}-\mathbf{x}_{0}^{*}\right\| \leqslant \alpha$, then for all $t \geqslant t_{0}$

$$
\left\|\mathbf{x}\left(t ; t_{0}, x_{0}\right)-\mathbf{x}^{*}\left(t ; t_{0}, x_{0}^{*}\right)\right\|<\gamma\left\|\mathbf{x}_{0}-\mathbf{x}_{0}^{*}\right\| \exp \left\{-\varphi\left(t-t_{0}\right)\right\}
$$

Definition 4.2 (Ahmad and Stamova $[24,28]$ ). We say that the function $V(t, \mathbf{x}), V:\left[t_{0}, \infty\right) \times$ $\mathbb{R}_{+}^{n+2}$ belongs to the class $V_{0}$ if the following conditions are satisfied:

(1) the function $V$ is continuous in $\bigcup_{i=1}^{\infty} G_{i}$, and $V(t, 0)=0$ for $t \in\left[t_{0}, \infty\right)$;

(2) the function $V$ satisfies locally the Lipschitz condition with respect to $\mathbf{x}$ on each of the sets $G_{i}$;

(3) for each $k \in \mathbb{Z}^{+}$, there exist the finite limits:

$$
V\left(t_{k}-0, \mathbf{x}\right)=\lim _{t \rightarrow t_{k}, t<t_{k}} V(t, \mathbf{x}), \quad V\left(t_{k}+0, \mathbf{x}\right)=\lim _{t \rightarrow t_{k}, t>t_{k}} V(t, \mathbf{x})
$$

(4) for each $k \in \mathbb{Z}^{+}$, the following equalities are valid:

$$
V\left(t_{k-1}, \mathbf{x}\right)=V\left(t_{k}, \mathbf{x}\right)
$$

In the proofs of the main theorems, we will use the following comparison results.

Lemma 4.3. Suppose the hypotheses (A1)-(A5) hold. There exist functions $P_{i}, Q_{i}, \widetilde{P}_{j}, \widetilde{Q}_{j} \in \mathbb{R}^{n+2}$ such that $P_{i}(t) \leqslant x_{i}(t) \leqslant Q_{i}(t), \widetilde{P}_{j}(t) \leqslant y_{j}(t) \leqslant \widetilde{Q}_{j}(t)$ for all $t \geqslant t_{0}$.

Proof. First we will prove that

$$
x_{i}(t) \leqslant Q_{i}(t), \quad y_{j}(t) \leqslant \widetilde{Q}_{j}(t),
$$


for all $t \geqslant t_{0}$, where $\left(Q_{i}(t), \widetilde{Q}_{j}(t)\right)=\left(q_{1}(t), q_{2}(t), \widetilde{q}_{1}(t), \ldots, \widetilde{q}_{n}(t)\right)$ is the maximal solution of the system:

$$
\begin{gathered}
\dot{q}_{i}(t)=q_{i}(t)\left(b_{i}(t)-a_{i}(t) q_{i}(t)\right), \\
\dot{\tilde{q}}_{j}(t)=\tilde{q}_{j}(t)\left(-r_{j}(t)+\sum_{k=1}^{2} \frac{d_{j k}(t) \tilde{q}_{k}(t)}{\alpha_{j k}(t)+\beta_{j k}(t) \tilde{q}_{k}(t)+\gamma_{j k}(t) \tilde{q}_{j}(t)}\right), \\
t \neq t_{k}, \quad\left(k \in \mathbb{Z}^{+}\right), \\
q_{i}\left(t_{0}^{+}\right)=q_{i 0}>0, \quad \tilde{q}_{j}\left(t_{0}^{+}\right)=\tilde{q}_{j 0}>0, \\
q_{i}\left(t_{k}^{+}\right)=\left(1+b_{i k}\right) q_{i}\left(t_{k}\right), \\
\tilde{q}_{j}\left(t_{k}^{+}\right)=\left(1+\tilde{b}_{j k}\right) \tilde{q}_{j}\left(t_{k}\right), \\
t=t_{k}, \quad\left(k \in \mathbb{Z}^{+}\right) .
\end{gathered}
$$

The maximal solution

$$
Q_{i}(t)=Q_{i}\left(t ; t_{0}, q_{0}\right), \quad \tilde{Q}_{j}(t)=Q_{i}\left(t ; t_{0}, q_{0}\right), \quad q_{0}=\left(q_{10}, q_{20}, \tilde{q}_{30}, \ldots, \tilde{q}_{(n+2) 0}\right)
$$

of (4.10) is defined by the equality:

$$
\begin{aligned}
& Q_{i}\left(t ; t_{0}, q_{0}\right)=\left\{\begin{array}{cc}
Q_{i}^{0}\left(t ; t_{0}, Q_{i}^{0}+0\right), & t_{0}<t \leqslant t_{1}, \\
Q_{i}^{1}\left(t ; t_{1}, Q_{i}^{1}+0\right), & t_{1}<t \leqslant t_{2}, \\
\ldots, & \\
Q_{i}^{k}\left(t ; t_{k}, Q_{i}^{k}+0\right), & t_{k}<t \leqslant t_{k+1}, \\
\ldots, &
\end{array}\right. \\
& \widetilde{Q}_{j}\left(t ; t_{0}, q_{0}\right)=\left\{\begin{array}{cc}
\widetilde{Q}_{j}^{0}\left(t ; t_{0}, \widetilde{Q}_{j}^{0}+0\right), & t_{0}<t \leqslant t_{1}, \\
\tilde{Q}_{j}^{1}\left(t ; t_{1}, \widetilde{Q}_{j}^{1}+0\right), & t_{1}<t \leqslant t_{2}, \\
\ldots, & \\
\tilde{Q}_{j}^{k}\left(t ; t_{k}, \widetilde{Q}_{j}^{k}+0\right), & t_{k}<t \leqslant t_{k+1}, \\
\ldots, &
\end{array}\right.
\end{aligned}
$$

where $Q_{k}^{i}\left(t ; t_{k}, Q_{i}^{k}+0\right), \widetilde{Q}_{k}^{j}\left(t ; t_{k}, \widetilde{Q}_{j}^{k}+0\right)$ is the solution of the equation without impulses

$$
\begin{gathered}
\dot{q}_{i}(t)=q_{i}(t)\left(b_{i}(t)-a_{i}(t) q_{i}(t)\right), \\
\dot{\tilde{q}}_{j}(t)=\tilde{q}_{j}(t)\left(-r_{j}(t)+\sum_{k=1}^{2} \frac{d_{j k}(t) q_{k}(t)}{\alpha_{j k}(t)+\beta_{j k}(t) q_{k}(t)+\gamma_{j k}(t) \tilde{q}_{j}(t)}\right)
\end{gathered}
$$


in the interval $\left(t_{k}, t_{k+1}\right], k \in \mathbb{Z}^{+}$, for which $Q_{i}^{k}+0=\left(1+b_{i k}\right) Q_{i}^{k}\left(t_{k} ; t_{k-1}, Q_{i}^{k-1}+0\right), \widetilde{Q}_{j}^{k}+0=\left(1+\tilde{b}_{j k}\right)$, $\widetilde{Q}_{j}^{k}\left(t_{k} ; t_{k-1}, \widetilde{Q}_{j}^{k-1}+0\right), k \in \mathbb{Z}^{+}$, and $Q_{i}^{0}+0=q_{i 0}, \widetilde{Q}_{j}^{0}+0=\tilde{q}_{j 0}$.

We note that the solutions $x_{i}(t), y_{j}(t)$ of (1.1) are functions which for $t \neq t_{k}, k \in \mathbb{Z}^{+}$ satisfy

$$
\begin{array}{r}
\dot{x}_{1}(t)=x_{1}(t)\left(b_{1}(t)-a_{1}(t) x_{1}(t)-\frac{x_{1}(t)}{\mu_{1}(t)+\omega_{1}(t) x_{2}(t)}\right. \\
\left.\quad-\sum_{k=1}^{n} \frac{c_{1 k}(t) y_{k}(t)}{\alpha_{1 k}(t)+\beta_{1 k}(t) x_{1}(t)+\gamma_{1 k}(t) y_{k}(t)}\right), \\
\dot{x}_{2}(t)=x_{2}(t)\left(b_{2}(t)-a_{2}(t) x_{2}(t)-\frac{x_{2}(t)}{\mu_{2}(t)+\omega_{2}(t) x_{1}(t)}\right. \\
\left.\quad-\sum_{k=1}^{n} \frac{c_{2 k}(t) y_{k}(t)}{\alpha_{2 k}(t)+\beta_{2 k}(t) x_{2}(t)+\gamma_{2 k}(t) y_{k}(t)}\right), \\
\dot{y}_{j}(t)=y_{j}(t)\left(-r_{j}(t)+\sum_{k=1}^{2} \frac{d_{j k}(t) x_{k}(t)}{\alpha_{j k}(t)+\beta_{j k}(t) x_{k}(t)+\gamma_{j k}(t) y_{j}(t)}\right. \\
\left.-\sum_{k=1}^{n} \delta_{j k}(t) y_{k}(t)\right),
\end{array}
$$

and for $t=t_{k}, k \in \mathbb{Z}^{+}$, satisfying the conditions $x_{i}\left(t_{k}^{+}\right)=\left(1+b_{i k}\right) x_{i}\left(t_{k}\right), y_{j}\left(t_{k}^{+}\right)=\left(1+\tilde{b}_{j k}\right) y_{j}\left(t_{k}\right)$, By hypothesis (A1), it follows from (1.1) that

$$
\begin{gathered}
\dot{x}_{i}(t) \leqslant x_{i}(t)\left(b_{i}(t)-a_{i}(t) x_{i}(t)\right), \\
\dot{y}_{j}(t) \leqslant y_{j}(t)\left(-r_{j}(t)+\sum_{k=1}^{2} \frac{d_{j k}(t) q_{k}(t)}{\alpha_{j k}(t)+\beta_{j k}(t) q_{k}(t)+\gamma_{j k}(t) \tilde{q}_{j}(t)}\right),
\end{gathered}
$$

for $t \neq t_{k}$. The elementary differential inequality (4.15) yields that

$$
x_{i}(t) \leqslant Q_{i}(t), \quad y_{j}(t) \leqslant \widetilde{Q}_{j}(t),
$$

for all $t \in\left(t_{0}, t_{1}\right]$, that is, the inequality (4.9) is valid for $t \in\left(t_{0}, t_{1}\right]$. Suppose that (4.9) is satisfied for $t \in\left(t_{k-1}, t_{k}\right], k>1$. Then using hypotheses (A2)-(A4) and the fact that (4.9) is satisfied for $t=t_{k}$, we obtain

$$
\begin{gathered}
x_{i}\left(t_{k}^{+}\right)=\left(1+b_{i k}\right) x_{i}\left(t_{k}\right) \leqslant\left(1+b_{i k}\right) Q_{i}\left(t_{k}\right)=Q_{i}\left(t_{k}^{+}\right), \\
y_{j}\left(t_{k}^{+}\right)=\left(1+\tilde{b}_{j k}\right) y_{j}\left(t_{k}\right) \leqslant\left(1+\tilde{b}_{j k}\right) \widetilde{Q}_{j}\left(t_{k}\right)=\widetilde{Q}_{j}\left(t_{k}^{+}\right) .
\end{gathered}
$$


We apply again the comparison result (4.15) in the interval $\left(t_{k}, t_{k+1}\right]$ and obtain

$$
\begin{aligned}
& x_{i}\left(t ; t_{0}, x_{i 0}\right) \leqslant Q_{i}^{k}\left(t ; t_{k}, Q_{i}^{k}+0\right)=Q_{i}\left(t ; t_{0}, q_{0}\right), \\
& y_{j}\left(t ; t_{0}, y_{j 0}\right) \leqslant \widetilde{Q}_{j}^{k}\left(t ; t_{k}, \widetilde{Q}_{j}^{k}+0\right)=\widetilde{Q}_{j}\left(t ; t_{0}, q_{0}\right),
\end{aligned}
$$

that is, the inequality (4.9) is valid for $\left(t_{k}, t_{k+1}\right]$. The proof of (4.9) is completed by induction. Further, by analogous arguments, using (A1)-(A4), we obtain from (1.1) and (4.15) the following:

$$
\begin{gathered}
\dot{x}_{1}(t) \geqslant x_{1}(t)\left(b_{1}(t)-a_{1}(t) x_{1}(t)-\frac{Q_{1}(t)}{\mu_{1}(t)+\omega_{1}(t) x_{2}(t)}\right. \\
\left.-\sum_{k=1}^{n} \frac{c_{1 k}(t) \widetilde{Q}_{k}(t)}{\alpha_{1 k}(t)+\beta_{1 k}(t) x_{1}(t)+\gamma_{1 k}(t) y_{k}(t)}\right), \\
\dot{x}_{2}(t) \geqslant x_{2}(t)\left(b_{2}(t)-a_{2}(t) x_{2}(t)-\frac{Q_{2}(t)}{\mu_{2}(t)+\omega_{2}(t) x_{1}(t)}\right. \\
\dot{y}_{j}(t) \geqslant y_{j}(t)\left(-\sum_{k=1}^{n} \frac{c_{2 k}(t) \widetilde{Q}_{k}(t)}{\alpha_{2 k}(t)+\beta_{2 k}(t) x_{1}(t)+\gamma_{2 k}(t) y_{k}(t)}\right), \\
-\sum_{k=1}^{n} \frac{d_{j k}(t) x_{k}(t)}{\alpha_{j k}(t)+\beta_{j k}(t) x_{k}(t)+\gamma_{j k}(t) y_{j}(t)} \\
\left.\delta_{j k}(t) \widetilde{Q}_{k}(t)\right), \\
t \neq t_{k}, \quad\left(k \in \mathbb{Z}^{+}\right), \\
x_{i}\left(t_{k}^{+}\right)=\left(1+b_{i k}\right) x_{i}\left(t_{k}\right), \\
y_{j}\left(t_{k}^{+}\right)=\left(1+\tilde{b}_{j k}\right) y_{j}\left(t_{k}\right), \\
t=t_{k \prime} \quad\left(k \in \mathbb{Z}^{+}\right),
\end{gathered}
$$

and hence

$$
x_{i}(t) \geqslant P_{i}(t), \quad y_{j}(t) \geqslant \widetilde{P}_{j}(t)
$$


for all $t \geqslant t_{0}$, where $\left(P_{i}(t), \tilde{P}_{j}(t)\right)=\left(p_{1}(t), p_{2}(t), \tilde{p}_{1}(t), \ldots, \tilde{p}_{n}(t)\right)$ is the minimal solution of the system:

$$
\begin{gathered}
\dot{p}_{1}(t)=p_{1}(t)\left(b_{1}(t)-a_{1}(t) x_{1}(t)-\frac{Q_{1}(t)}{\mu_{1}(t)+\omega_{1}(t) x_{2}(t)}\right. \\
\left.-\sum_{k=1}^{n} \frac{c_{1 k}(t) \widetilde{Q}_{k}(t)}{\alpha_{1 k}(t)+\beta_{1 k}(t) x_{1}(t)+\gamma_{1 k}(t) y_{k}(t)}\right), \\
\dot{p}_{2}(t)=p_{2}(t)\left(b_{2}(t)-a_{2}(t) x_{2}(t)-\frac{Q_{2}(t)}{\mu_{2}(t)+\omega_{2}(t) x_{1}(t)}\right. \\
\left.-\sum_{k=1}^{n} \frac{c_{2 k}(t) \widetilde{Q}_{k}(t)}{\alpha_{2 k}(t)+\beta_{2 k}(t) x_{1}(t)+\gamma_{2 k}(t) y_{k}(t)}\right), \\
\dot{\tilde{p}}_{j}(t)=\tilde{p}_{j}(t)\left(\begin{array}{c}
-r_{j}(t)+\sum_{k=1}^{2} \frac{d_{j k}(t) p_{k}(t)}{\alpha_{j k}(t)+\beta_{j k}(t) p_{k}(t)+\gamma_{j k}(t) \tilde{p}_{j}(t)} \\
\left.-\sum_{k=1}^{n} \delta_{j k}(t) \tilde{Q}_{k}(t)\right), \\
t \neq t_{k}, \quad\left(k \in \mathbb{Z}^{+}\right), \\
p_{i}\left(t_{0}^{+}\right)=p_{i 0}>0, \quad \tilde{p}_{j}\left(t_{0}^{+}\right)=\tilde{p}_{j 0}>0, \\
p_{i}\left(t_{k}^{+}\right)=\left(1+b_{i k}\right) p_{i}\left(t_{k}\right), \\
\tilde{p}_{j}\left(t_{k}^{+}\right)=\left(1+\tilde{b}_{j k}\right) \tilde{p}_{j}\left(t_{k}\right), \\
t=t_{k}, \quad\left(k \in \mathbb{Z}^{+}\right) .
\end{array}\right.
\end{gathered}
$$

Thus, the proof follows from (4.9) and (4.20).

Lemma 4.4. Suppose the hypotheses (A1)-(A5) hold. $\mathbf{x}(t)=\mathbf{x}\left(t ; t_{0}, x_{0}\right)=$ $\left(x_{1}(t), x_{2}(t), y_{1}(t), \ldots, y_{n}(t)\right)$ is a solution of (1.1), then there exist positive constants: $\alpha_{i}, \beta_{i}, \tilde{\alpha}_{j}$, and $\tilde{\beta}_{j}$ such that

$$
\alpha_{i} \leqslant x_{i}(t) \leqslant \beta_{i}, \quad \tilde{\alpha}_{j} \leqslant y_{j}(t) \leqslant \tilde{\beta}_{j}
$$

for all $t \in\left(t_{k-1}, t_{k}\right], k \in \mathbb{Z}^{+}$and if in addition

$$
0<1+b_{i k} \leqslant 1, \quad 0<1+\tilde{b}_{j k} \leqslant 1,
$$

then

$$
\alpha_{i} \leqslant x_{i}(t) \leqslant \beta_{i}, \quad \tilde{\alpha}_{j} \leqslant y_{j}(t) \leqslant \tilde{\beta}_{j}
$$

for all $t \in J$. 
Proof. From Lemma 4.3, we have $P_{i}(t) \leqslant x_{i}(t) \leqslant Q_{i}(t), \widetilde{P}_{j}(t) \leqslant y_{j}(t) \leqslant \widetilde{Q}_{j}(t)$ for all $t \geqslant t_{0}$, where $P_{i}(t), \widetilde{P}_{j}(t)$ is the minimal solution of the system $(4.21)$, and $Q_{i}(t), \widetilde{Q}_{j}(t)$ is the maximal solution of the system (4.10). Under the conditions of Lemma 4.4 for the solutions of (4.10) and (4.21) with initial functions, it is valid that

$$
\alpha_{i} \leqslant P_{i}(t), \quad Q_{i}(t) \leqslant \beta_{i}, \quad \tilde{\alpha}_{j} \leqslant \widetilde{P}_{j}(t), \quad \widetilde{Q}_{j}(t) \leqslant \tilde{\beta}_{j},
$$

$\alpha_{i}, \tilde{\alpha}_{j}>0,0<\beta_{i}, \tilde{\beta}_{j}<\infty$, for all $t \geqslant t_{0}, t \in\left(t_{k-1}, t_{k}\right], k \in \mathbb{Z}^{+}$, then $\alpha_{i} \leqslant x_{i}(t) \leqslant \beta_{i}, \tilde{\alpha}_{j} \leqslant y_{j}(t) \leqslant \tilde{\beta}_{j}$, for all $t \geqslant t_{0}, t \in\left(t_{k-1}, t_{k}\right], k \in \mathbb{Z}^{+}$. If in addition $0<1+b_{i k} \leqslant 1,0<1+\widetilde{b}_{j k} \leqslant 1$, then from the left continuity of $x_{i}(t)$ at the points $t_{k}$, we have

$$
\begin{aligned}
\alpha_{i} & =\left(1+b_{i k}\right) \alpha_{i}-\left(b_{i k}\right) \alpha_{i} \leqslant\left(1+b_{i k}\right) x_{i}(t)-\left(b_{i k}\right) x_{i}(t) \\
& \leqslant\left(1+b_{i k}\right) \beta_{i}-\left(b_{i k}\right) \beta_{i}=\beta_{i}, \\
\tilde{\alpha}_{j} & =\left(1+\tilde{b}_{j k}\right) \tilde{\alpha}_{j}-\left(\tilde{b}_{j k}\right) \tilde{\alpha}_{j} \leqslant\left(1+\widetilde{b}_{j k}\right) y_{j}(t)-\left(\tilde{b}_{j k}\right) y_{j}(t) \\
& \leqslant\left(1+\tilde{b}_{j k}\right) \tilde{\beta}_{j}-\left(\tilde{b}_{j k}\right) \tilde{\beta}_{j}=\tilde{\beta}_{j},
\end{aligned}
$$

that is,

$$
\alpha_{i} \leqslant x_{j}\left(t_{k}^{+}\right) \leqslant \beta_{i}, \quad \tilde{\alpha}_{j} \leqslant y_{j}\left(t_{k}^{+}\right) \leqslant \tilde{\beta}_{j}
$$

hence

$$
\alpha_{i} \leqslant x_{i}(t) \leqslant \beta_{i}, \quad \tilde{\alpha}_{j} \leqslant y_{j}(t) \leqslant \tilde{\beta}_{j}
$$

for all $t \geqslant t_{0}$.

Let $V \in V_{0}$, for any $(t, \mathbf{x}) \in\left[t_{k-1}, t_{k}\right) \times \mathbb{R}_{+}^{n+2}$, the right-hand derivative $D^{+} V(t, \mathbf{x}(t))$ along the solution $\mathbf{x}\left(t, t_{0}, x_{0}\right)$ of $(1.1)$ is defined by

$$
D^{+} V(t, \mathbf{x}(t))=\lim _{h \rightarrow 0^{+}} \inf \frac{1}{h}[V(t+h, \mathbf{x}(t+h))-V(t, \mathbf{x}(t))] .
$$

Define

$$
m(t)=\sum_{i=1}^{2}\left|x_{i}(t)-x_{i}^{*}(t)\right|+\sum_{j=1}^{n}\left|y_{j}(t)-y_{j}^{*}(t)\right|
$$

and consider a Lyapunov function:

$$
V\left(\mathbf{x}(t), \mathbf{x}^{*}(t)\right)=\sum_{i=1}^{n+2} V_{i}(t)=\sum_{i=1}^{2}\left|\ln \frac{x_{i}(t)}{x_{i}^{*}(t)}\right|+\sum_{j=1}^{n}\left|\ln \frac{y_{j}(t)}{y_{j}^{*}(t)}\right|
$$


Theorem 4.5. Let the following conditions hold:

(1) the hypotheses (A1)-(A5) hold;

(2) there exist nonnegative continuous functions $\delta_{i}(t)$, and $\delta_{j}(t)$ such that

$$
\begin{aligned}
a_{1}^{\ell}+ & \frac{\mu_{1}^{\ell}+\omega_{1}^{\ell} \alpha_{2}^{*}}{\left(\mu_{1}^{u}+\omega_{1}^{u} \beta_{2}\right)\left(\mu_{1}^{u}+\omega_{1}^{u} \beta_{2}^{*}\right)} \\
> & \sum_{k=1}^{n} \frac{c_{1 k}^{u} \beta_{1 k}^{u} \tilde{\beta}_{k}}{\left(\alpha_{1 k}^{\ell}+\beta_{1 k}^{\ell} \alpha_{1}+\gamma_{1 k}^{\ell} \tilde{\alpha}_{k}\right)\left(\alpha_{1 k}^{\ell}+\beta_{1 k}^{\ell} \alpha_{1}^{*}+\gamma_{1 k}^{\ell} \tilde{\alpha}_{k}^{*}\right)}+\frac{\omega_{2}^{u} \beta_{1}^{*}}{\left(\mu_{2}^{\ell}+\omega_{2}^{\ell} \alpha_{1}\right)\left(\mu_{2}^{\ell}+\omega_{2}^{\ell} \alpha_{1}^{*}\right)} \\
& +\sum_{j=1}^{n} \frac{d_{j 1}^{u}\left(\alpha_{j 1}^{u}+\gamma_{j 1}^{u} \tilde{\beta}_{j}\right)}{\left(\alpha_{j 1}^{\ell}+\beta_{j 1}^{\ell} \alpha_{1}+\gamma_{j 1}^{\ell} \tilde{\alpha}_{j}\right)\left(\alpha_{j 1}^{\ell}+\beta_{j 1}^{\ell} \alpha_{1}^{*}+\gamma_{j 1}^{\ell} \tilde{\alpha}_{j}^{*}\right)}, \\
a_{2}^{\ell}+ & \frac{\mu_{2}^{\ell}+\omega_{2}^{\ell} \alpha_{1}^{*}}{\left(\mu_{2}^{u}+\omega_{1}^{u} \beta_{1}\right)\left(\mu_{2}^{u}+\omega_{2}^{u} \beta_{1}^{*}\right)} \\
> & \sum_{k=1}^{n} \frac{c_{2 k}^{u} \beta_{2 k}^{u} \tilde{\beta}_{k}}{\left(\alpha_{2 k}^{\ell}+\beta_{2 k}^{\ell} \alpha_{2}+\gamma_{2 k}^{\ell} \tilde{\alpha}_{k}\right)\left(\alpha_{2 k}^{\ell}+\beta_{2 k}^{\ell} \alpha_{2}^{*}+\gamma_{2 k}^{\ell} \tilde{\alpha}_{k}^{*}\right)}+\frac{\omega_{1}^{u} \beta_{2}^{*}}{\left(\mu_{1}^{\ell}+\omega_{1}^{\ell} \alpha_{2}\right)\left(\mu_{1}^{\ell}+\omega_{1}^{\ell} \alpha_{2}^{*}\right)} \\
& +\sum_{j=1}^{n} \frac{d_{j 2}^{u}\left(\alpha_{j 2}^{u}+\gamma_{j 2}^{u} \tilde{\beta}_{j}\right)}{\left(\alpha_{j 2}^{\ell}+\beta_{j 2}^{\ell} \alpha_{2}+\gamma_{j 2}^{\ell} \tilde{\alpha}_{j}\right)\left(\alpha_{j 2}^{\ell}+\beta_{j 2}^{\ell} \alpha_{2}^{*}+\gamma_{j 2}^{\ell} \tilde{\alpha}_{j}^{*}\right)}, \\
\delta_{j j}^{\ell}+\sum_{k=1}^{2} & \frac{d_{j k}^{\ell} \gamma_{j k}^{\ell} \alpha_{k}}{\left(\alpha_{j k}^{u}+\beta_{j k}^{u} \beta_{k}+\gamma_{j k}^{u} \tilde{\beta}_{j}\right)\left(\alpha_{j k}^{u}+\beta_{j k}^{u} \beta_{k}^{*}+\gamma_{j k}^{u} \tilde{\beta}_{j}^{*}\right)} \\
& \sum_{k=1, k \neq j}^{n} \delta_{j k}^{u}+\sum_{i=1}^{2} \frac{c_{i j}^{u}\left(\alpha_{i j}^{u}+\beta_{i j}^{u} \beta_{i}\right)}{\left(\alpha_{i j}^{\ell}+\beta_{i j}^{\ell} \alpha_{i}+\gamma_{i j}^{\ell} \tilde{\alpha}_{j}\right)\left(\alpha_{i j}^{\ell}+\beta_{i j}^{\ell} \alpha_{i}^{*}+\gamma_{i j}^{\ell} \tilde{\alpha}_{j}^{*}\right)}
\end{aligned}
$$

(3) $0<1+b_{i k} \leqslant 1,0<1+\tilde{b}_{j k} \leqslant 1$.

Then, the solution $x(t)$ of (1.1) is globally stable. 
Proof. Consider the upper right derivative $D^{+} V\left(\mathbf{x}(t), \mathbf{x}^{*}(t)\right)$ along the solution of system (1.1). For $t \geqslant t_{0}$ and $t \neq t_{k}, k \in \mathbb{Z}^{+}$, we derive the estimate as follows:

$$
\begin{aligned}
& D^{+} V\left(\mathbf{x}(t), \mathbf{x}^{*}(t)\right) \\
& =\left(-a_{1}(t)\left(x_{k}(t)-x_{k}^{*}(t)\right)-\sum_{k=1}^{n}\left(\frac{c_{1 k}(t) y_{k}(t)}{\alpha_{1 k}(t)+\beta_{1 k}(t) x_{1}(t)+\gamma_{1 k}(t) y_{k}(t)}\right.\right. \\
& \left.-\frac{c_{1 k}(t) y_{k}^{*}(t)}{\alpha_{1 k}(t)+\beta_{1 k}(t) x_{1}^{*}(t)+\gamma_{1 k}(t) y_{k}^{*}(t)}\right) \\
& \left.-\left(\frac{x_{1}(t)}{\mu_{1}(t)+\omega_{1}(t) x_{2}(t)}-\frac{x_{1}^{*}(t)}{\mu_{1}(t)+\omega_{1}(t) x_{2}^{*}(t)}\right)\right) \\
& \times \operatorname{sgn}\left(x_{1}-x_{1}^{*}\right)+\left(-a_{2}(t)\left(x_{2}(t)-x_{2}^{*}(t)\right)\right. \\
& -\sum_{k=1}^{n}\left(\frac{c_{2 k}(t) y_{k}(t)}{\alpha_{2 k}(t)+\beta_{2 k}(t) x_{2}(t)+\gamma_{2 k}(t) y_{k}(t)}\right. \\
& \left.-\frac{c_{2 k}(t) y_{k}^{*}(t)}{\alpha_{2 k}(t)+\beta_{2 k}(t) x_{2}^{*}(t)+\gamma_{2 k}(t) y_{k}^{*}(t)}\right) \\
& \left.-\left(\frac{x_{2}(t)}{\mu_{2}(t)+\omega_{2}(t) x_{1}(t)}-\frac{x_{2}^{*}(t)}{\mu_{2}(t)+\omega_{2}(t) x_{1}^{*}(t)}\right)\right) \\
& \times \operatorname{sgn}\left(x_{2}-x_{2}^{*}\right) \\
& +\sum_{j=1}^{n}\left(\sum_{k=1}^{2}\left(\frac{d_{j k}(t) x_{k}(t)}{\alpha_{j k}(t)+\beta_{j k}(t) x_{k}(t)+\gamma_{j k}(t) y_{j}(t)}-\frac{d_{j k}(t) x_{k}^{*}(t)}{\alpha_{j k}(t)+\beta_{j k}(t) x_{k}^{*}(t)+\gamma_{j k}(t) y_{j}^{*}(t)}\right)\right. \\
& \left.-\sum_{k=1}^{n} \delta_{j k}(t)\left(y_{k}(t)-y_{k}^{*}(t)\right)\right) \operatorname{sgn}\left(y_{j}-y_{j}^{*}\right) \\
& \leqslant \sum_{i=1}^{2}\left(-a_{i}^{\ell}\left|x_{i}(t)-x_{i}^{*}(t)\right|+\sum_{k=1}^{n} \frac{c_{i k}^{u} \alpha_{i k}^{u}}{\left(\alpha_{i k}^{\ell}+\beta_{i k}^{\ell} x_{i}(t)+\gamma_{i k}^{\ell} y_{k}(t)\right)\left(\alpha_{i k}^{\ell}+\beta_{i k}^{\ell} x_{i}^{*}(t)+\gamma_{i k}^{\ell} y_{k}^{*}(t)\right)}\right. \\
& \times\left|y_{k}(t)-y_{k}^{*}(t)\right| \\
& +\sum_{k=1}^{n} \frac{c_{i k}^{u} \beta_{i k}^{u} y_{k}(t)}{\left(\alpha_{i k}^{\ell}+\beta_{i k}^{\ell} x_{i}(t)+\gamma_{i k}^{\ell} y_{k}(t)\right)\left(\alpha_{i k}^{\ell}+\beta_{i k}^{\ell} x_{i}^{*}(t)+\gamma_{i k}^{\ell} y_{k}^{*}(t)\right)}\left|x_{i}(t)-x_{i}^{*}(t)\right| \\
& \left.+\sum_{k=1}^{n} \frac{c_{i k}^{u} \beta_{i k}^{u} x_{i}(t)}{\left(\alpha_{i k}^{\ell}+\beta_{i k}^{\ell} x_{i}(t)+\gamma_{i k}^{\ell} y_{k}(t)\right)\left(\alpha_{i k}^{\ell}+\beta_{i k}^{\ell} x_{i}^{*}(t)+\gamma_{i k}^{\ell} y_{k}^{*}(t)\right)}\left|y_{k}(t)-y_{k}^{*}(t)\right|\right)
\end{aligned}
$$


Abstract and Applied Analysis

29

$$
\begin{aligned}
& -\frac{\mu_{1}^{\ell}+\omega_{1}^{\ell} x_{2}^{*}(t)}{\left(\mu_{1}^{u}+\omega_{1}^{u} x_{2}(t)\right)\left(\mu_{1}^{u}+\omega_{1}^{u} x_{2}^{*}(t)\right)}\left|x_{1}(t)-x_{1}^{*}(t)\right| \\
& +\frac{\omega_{1}^{u} x_{2}^{*}(t)}{\left(\mu_{1}^{\ell}+\omega_{1}^{\ell} x_{2}(t)\right)\left(\mu_{1}^{\ell}+\omega_{1}^{\ell} x_{2}^{*}(t)\right)}\left|x_{2}(t)-x_{2}^{*}(t)\right| \\
& -\frac{\mu_{2}^{\ell}+\omega_{2}^{\ell} x_{1}^{*}(t)}{\left(\mu_{2}^{u}+\omega_{2}^{u} x_{1}(t)\right)\left(\mu_{2}^{u}+\omega_{2}^{u} x_{1}^{*}(t)\right)}\left|x_{2}(t)-x_{2}^{*}(t)\right| \\
& +\frac{\omega_{2}^{u} x_{1}^{*}(t)}{\left(\mu_{2}^{\ell}+\omega_{2}^{\ell} x_{1}(t)\right)\left(\mu_{2}^{\ell}+\omega_{2}^{\ell} x_{1}^{*}(t)\right)}\left|x_{1}(t)-x_{1}^{*}(t)\right| \\
& +\sum_{j=1}^{n}\left(\sum_{k=1}^{2} \frac{d_{j k}^{u} \alpha_{j k}^{u}}{\left(\alpha_{j k}^{\ell}+\beta_{j k}^{\ell} x_{k}(t)+\gamma_{j k}^{\ell} y_{j}(t)\right)\left(\alpha_{j k}^{\ell}+\beta_{j k}^{\ell} x_{k}^{*}(t)+\gamma_{j k}^{\ell} y_{j}^{*}(t)\right)}\left|x_{k}(t)-x_{k}^{*}(t)\right|\right. \\
& +\sum_{k=1}^{2} \frac{d_{j k}^{u} \gamma_{j k}^{u} y_{j}(t)}{\left(\alpha_{j k}^{\ell}+\beta_{j k}^{\ell} x_{k}(t)+\gamma_{j k}^{\ell} y_{j}(t)\right)\left(\alpha_{j k}^{\ell}+\beta_{j k}^{\ell} x_{k}^{*}(t)+\gamma_{j k}^{\ell} y_{j}^{*}(t)\right)}\left|x_{k}(t)-x_{k}^{*}(t)\right| \\
& -\sum_{k=1}^{2} \frac{d_{j k}^{\ell} \gamma_{j k}^{\ell} x_{k}(t)}{\left(\alpha_{j k}^{u}+\beta_{j k}^{u} x_{k}(t)+\gamma_{j k}^{u} y_{j}(t)\right)\left(\alpha_{j k}^{u}+\beta_{j k}^{u} x_{k}^{*}(t)+\gamma_{j k}^{u} y_{j}^{*}(t)\right)}\left|y_{j}(t)-y_{j}^{*}(t)\right| \\
& \left.-\delta_{j j}^{\ell}\left|y_{j}-y_{j}^{*}\right|+\sum_{k=1, k \neq j}^{n} \delta_{j k}^{u}\left|y_{k}(t)-y_{k}^{*}\right|\right) \\
& =\left(-a_{1}^{\ell}+\frac{\mu_{1}^{\ell}+\omega_{1}^{\ell} \alpha_{2}^{*}}{\left(\mu_{1}^{u}+\omega_{1}^{u} \beta_{2}\right)\left(\mu_{1}^{u}+\omega_{1}^{u} \beta_{2}^{*}\right)}-\sum_{k=1}^{n} \frac{c_{1 k}^{u} \beta_{1 k}^{u} \tilde{\beta}_{k}}{\left(\alpha_{1 k}^{\ell}+\beta_{1 k}^{\ell} \alpha_{1}+\gamma_{1 k}^{\ell} \tilde{\alpha}_{k}\right)\left(\alpha_{1 k}^{\ell}+\beta_{1 k}^{\ell} \alpha_{1}^{*}+\gamma_{1 k}^{\ell} \tilde{\alpha}_{k}^{*}\right)}\right. \\
& \left.-\frac{\omega_{2}^{u} \beta_{1}^{*}}{\left(\mu_{2}^{\ell}+\omega_{2}^{\ell} \alpha_{1}\right)\left(\mu_{2}^{\ell}+\omega_{2}^{\ell} \alpha_{1}^{*}\right)}-\sum_{j=1}^{n} \frac{d_{j 1}^{u}\left(\alpha_{j 1}^{u}+\gamma_{j 1}^{u} \tilde{\beta}_{j}\right)}{\left(\alpha_{j 1}^{\ell}+\beta_{j 1}^{\ell} \alpha_{1}+\gamma_{j 1}^{\ell} \tilde{\alpha}_{j}\right)\left(\alpha_{j 1}^{\ell}+\beta_{j 1}^{\ell} \alpha_{1}^{*}+\gamma_{j 1}^{\ell} \tilde{\alpha}_{j}^{*}\right)}\right) \\
& \times\left|x_{1}(t)-x_{1}^{*}(t)\right|-\left(a_{2}^{\ell}+\frac{\mu_{2}^{\ell}+\omega_{2}^{\ell} \alpha_{1}^{*}}{\left(\mu_{2}^{u}+\omega_{1}^{u} \beta_{1}\right)\left(\mu_{2}^{u}+\omega_{2}^{u} \beta_{1}^{*}\right)}\right. \\
& -\sum_{k=1}^{n} \frac{c_{2 k}^{u} \beta_{2 k}^{u} \tilde{\beta}_{k}}{\left(\alpha_{2 k}^{\ell}+\beta_{2 k}^{\ell} \alpha_{2}+\gamma_{2 k}^{\ell} \widetilde{\alpha}_{k}\right)\left(\alpha_{2 k}^{\ell}+\beta_{2 k}^{\ell} \alpha_{2}^{*}+\gamma_{2 k}^{\ell} \tilde{\alpha}_{k}^{*}\right)} \\
& -\frac{\omega_{1}^{u} \beta_{2}^{*}}{\left(\mu_{1}^{\ell}+\omega_{1}^{\ell} \alpha_{2}\right)\left(\mu_{1}^{\ell}+\omega_{1}^{\ell} \alpha_{2}^{*}\right)} \\
& \left.-\sum_{j=1}^{n} \frac{d_{j 2}^{u}\left(\alpha_{j 2}^{u}+\gamma_{j 2}^{u} \tilde{\beta}_{j}\right)}{\left(\alpha_{j 2}^{\ell}+\beta_{j 2}^{\ell} \alpha_{2}+\gamma_{j 2}^{\ell} \tilde{\alpha}_{j}\right)\left(\alpha_{j 2}^{\ell}+\beta_{j 2}^{\ell} \alpha_{2}^{*}+\gamma_{j 2}^{\ell} \tilde{\alpha}_{j}^{*}\right)}\right)
\end{aligned}
$$




$$
\begin{aligned}
& \times\left|x_{2}(t)-x_{2}^{*}(t)\right| \\
& -\sum_{j=1}^{n}\left(\delta_{j j}^{\ell}+\sum_{k=1}^{2} \frac{d_{j k}^{\ell} \gamma_{j k}^{\ell} \alpha_{k}}{\left(\alpha_{j k}^{u}+\beta_{j k}^{u} \beta_{k}+\gamma_{j k}^{u} \tilde{\beta}_{j}\right)\left(\alpha_{j k}^{u}+\beta_{j k}^{u} \beta_{k}^{*}+\gamma_{j k}^{u} \widetilde{\beta}_{j}^{*}\right)}\right. \\
& \left.\quad-\sum_{k=1, k \neq j}^{n} \delta_{j k}^{u}-\sum_{i=1}^{2} \frac{c_{i j}^{u}\left(\alpha_{i j}^{u}+\beta_{i j}^{u} \beta_{i}\right)}{\left(\alpha_{i j}^{\ell}+\beta_{i j}^{\ell} \alpha_{i}+\gamma_{i j}^{\ell} \tilde{\alpha}_{j}\right)\left(\alpha_{i j}^{\ell}+\beta_{i j}^{\ell} \alpha_{i}^{*}+\gamma_{i j}^{\ell} \tilde{\alpha}_{j}^{*}\right)}\right) \\
& \times\left|y_{j}(t)-y_{j}^{*}\right| .
\end{aligned}
$$

Thus in view of hypothesis (A8), we obtain

$$
D^{+} V\left(\mathbf{x}(t), \mathbf{x}^{*}(t)\right) \leqslant-\delta(t) m(t), \quad t \geqslant t_{0}, t \neq t_{k},\left(k \in \mathbb{Z}^{+}\right),
$$

where $\delta(t)=\min \left\{\delta_{i}, \delta_{j}\right\}$.

For $t \geqslant t_{0}, t=t_{k},\left(k \in \mathbb{Z}^{+}\right)$, we have

$$
\begin{aligned}
V\left(\mathbf{x}\left(t_{k}^{+}\right), \mathbf{x}^{*}\left(t_{k}^{+}\right)\right) & =\sum_{i=1}^{2}\left|\ln \frac{x_{i}\left(t_{k}^{+}\right)}{x_{i}^{*}\left(t_{k}^{+}\right)}\right|+\sum_{j=1}^{n}\left|\ln \frac{y_{j}\left(t_{k}^{+}\right)}{y_{j}^{*}\left(t_{k}^{+}\right)}\right| \\
& =\sum_{i=1}^{2}\left|\ln \frac{\left(1+b_{i k}\right) x_{i}\left(t_{k}\right)}{\left(1+b_{i k}\right) x_{i}^{*}\left(t_{k}\right)}\right|+\sum_{j=1}^{n}\left|\ln \frac{\left(1+\tilde{b}_{j k}\right) y_{j}\left(\mathrm{t}_{k}\right)}{\left(1+\tilde{b}_{j k}\right) y_{j}^{*}\left(t_{k}\right)}\right| \\
& =V\left(\mathbf{x}\left(t_{k}\right), \mathbf{x}^{*}\left(t_{k}\right)\right) .
\end{aligned}
$$

Then the inequality,

$$
V\left(\mathbf{x}(t), \mathbf{x}^{*}(t)\right) \leqslant V\left(\mathbf{x}\left(t_{0}^{+}\right), \mathbf{x}^{*}\left(t_{0}^{+}\right)\right)-\int_{t_{0}}^{t} \delta(s) m(s) d s, \quad t \geqslant t_{0}
$$

holds.

By Mean Value Theorem and by Lemma 4.4 it follows that for any closed interval contained in $t \in\left(t_{k-1}, t_{k}\right], k \in \mathbb{Z}^{+}$, there exist positive numbers $r$ and $R$ such that for every $i, j, r \leqslant x_{i}(t), y_{j}(t), x_{i}^{*}(t), y_{j}^{*}(t) \leqslant R$ and

$$
\begin{aligned}
& \frac{1}{R}\left|x_{i}(t)-x_{i}^{*}(t)\right| \leqslant\left|\ln x_{i}(t)-\ln x_{i}^{*}(t)\right| \leqslant \frac{1}{r}\left|x_{i}(t)-x_{i}^{*}(t)\right| \\
& \frac{1}{R}\left|y_{j}(t)-y_{j}^{*}(t)\right| \leqslant\left|\ln y_{j}(t)-\ln y_{j}^{*}(t)\right| \leqslant \frac{1}{r}\left|y_{j}(t)-y_{j}^{*}(t)\right| .
\end{aligned}
$$


Hence, we obtain

$$
\begin{aligned}
V\left(\mathbf{x}_{0}, \mathbf{x}_{0}^{*}\right) & =\sum_{i=1}^{2}\left|\ln x_{i}\left(t_{0}^{+}\right)-\ln x_{i}^{*}\left(t_{0}^{+}\right)\right|+\sum_{j=1}^{n}\left|\ln y_{j}\left(t_{0}^{+}\right)-\ln y_{j}^{*}\left(t_{0}^{+}\right)\right| \\
& \leqslant \frac{1}{r}\left\|\mathbf{x}_{0}-\mathbf{x}_{0}^{*}\right\| .
\end{aligned}
$$

Further, from (4.34) and (4.35) we have

$$
\begin{gathered}
D^{+} V\left(\mathbf{x}(t), \mathbf{x}^{*}(t)\right) \leqslant 0, \quad t \geqslant t_{0}, \quad t \neq t_{k}, \\
\Delta V\left(\mathbf{x}\left(t_{k}\right), \mathbf{x}^{*}\left(t_{k}\right)\right)=0,
\end{gathered}
$$

and hence

$$
V\left(\mathbf{x}(t), \mathbf{x}^{*}(t)\right) \leqslant V\left(\mathbf{x}_{0}, \mathbf{x}_{0}^{*}\right)
$$

for all $t \geqslant t_{0}$. Given $0<\varepsilon<R$, choose $\delta=\varepsilon r / 2 R$. Then from (4.37)-(4.40) it follows that

$$
\sum_{i=1}^{2}\left|x_{i}(t)-x_{i}^{*}(t)\right|+\sum_{j=1}^{n}\left|y_{j}(t)-y_{j}^{*}(t)\right|<\varepsilon,
$$

for all $t \geqslant t_{0}$, whenever $\left\|\mathbf{x}_{0}-\mathbf{x}_{0}^{*}\right\| \leqslant \delta$ and $t_{0} \in \mathbb{R}_{+}$. Since $t_{0} \in \mathbb{R}_{+}$is arbitrary, by Definition 4.1(a), the system (1.1) is globally stable. This proves the theorem.

Theorem 4.6. In addition to the assumptions of Theorem 4.5, suppose that there exists a constant $c$ such that

$$
\int_{t_{0}}^{t} \delta(s) d s=c\left(t-t_{0}\right)
$$

Then the system (1.1) is globally exponentially stable.

Proof. We consider again the Lyapunov function (4.31). From (4.34) and (4.37), we obtain

$$
D^{+} V\left(\mathbf{x}(t), \mathbf{x}^{*}(t)\right) \leqslant-\delta(t) m(t) \leqslant-r \delta(t) V\left(\mathbf{x}(t), \mathbf{x}^{*}(t)\right)
$$

From the above mentioned estimate and (4.38), we have

$$
V\left(\mathbf{x}(t), \mathbf{x}^{*}(t)\right) \leqslant V\left(\mathbf{x}_{0}, \mathbf{x}_{0}^{*}\right) \exp \left\{-r \int_{t_{0}}^{t} \delta(s) d s\right\}
$$


for all $t \geqslant t_{0}$. Then from (4.37),(4.40), (4.44), and (4.38), we deduce the inequality

$$
\sum_{i=1}^{2}\left|x_{i}(t)-x_{i}^{*}(t)\right|+\sum_{j=1}^{n}\left|y_{j}(t)-y_{j}^{*}(t)\right| \leqslant \frac{R}{r}\left\|\mathbf{x}_{0}-\mathbf{x}_{0}^{*}\right\| e^{-r c\left(t-t_{0}\right)}
$$

for $t \geqslant t_{0}$. This shows that the system (1.1) is globally exponentially stable. This proves the theorem.

\section{Acknowledgment}

The second author was partially supported by the Project of Science and Technology of Heilongjiang Province of China no. 12521151.

\section{References}

[1] G. T. Skalski and J. F. Gilliam, "Functional responses with predator interference: viable alternatives to the Holling type II model," Ecology, vol. 82, no. 11, pp. 3083-3092, 2001.

[2] Z. Zeng, L. Bi, and M. Fan, "Existence of multiple positive periodic solutions for functional differential equations," Journal of Mathematical Analysis and Applications, vol. 325, no. 2, pp. 1378-1389, 2007.

[3] A. Hastings, "Multiple limit cycles in predator-prey models," Journal of Mathematical Biology, vol. 11, no. 1 , pp. 51-63, 1981.

[4] F. Brauer and A. C. Soudack, "Coexistence properties of some predator-prey systems under constant rate harvesting and stocking," Journal of Mathematical Biology, vol. 12, no. 1, pp. 101-114, 1982.

[5] M. Bohner, M. Fan, and J. Zhang, "Existence of periodic solutions in predator-prey and competition dynamic systems," Nonlinear Analysis: Real World Applications, vol. 7, no. 5, pp. 1193-1204, 2006.

[6] R. S. Cantrell and C. Cosner, "On the dynamics of predator-prey models with the BeddingtonDeAngelis functional response," Journal of Mathematical Analysis and Applications, vol. 257, no. 1, pp. 206-222, 2001.

[7] S. Froda and S. Nkurunziza, "Prediction of predator-prey populations modelled by perturbed ODEs," Journal of Mathematical Biology, vol. 54, no. 3, pp. 407-451, 2007.

[8] S. Froda and A. Zahedi, "Simple testing procedures for the Holling type II model," Theoretical Ecology, vol. 2, no. 3, pp. 149-160, 2009.

[9] M. R. Garvie and C. Trenchea, "Finite element approximation of spatially extended predator-prey interactions with the Holling type II functional response," Numerische Mathematik, vol. 107, no. 4, pp. 641-667, 2007.

[10] J. R. Beddington, "Mutual interference between parasites or predator and its effect on searching efficiency," Journal of Animal Ecology, vol. 44, pp. 331-340, 1975.

[11] D. L. DeAngelis, R. A. Goldstein, and R. V. Neill, “A model for trophic interaction,” Ecology, vol. 56, pp. 881-892, 1975.

[12] S. Sivasundaram and S. Vassilyev, "Stability and attractivity of solutions of differential equations with impulses at fixed times," Journal of Applied Mathematics and Stochastic Analysis, vol. 13, no. 1, pp. 77-84, 2000.

[13] V. Lakshmikantham, D. D. Bă̆nov, and P. S. Simeonov, Theory of Impulsive Differential Equations, vol. 6 of Series in Modern Applied Mathematics, World Scientific Publishing, Singapore, 1989.

[14] A. Anokhin, L. Berezansky, and E. Braverman, "Exponential stability of linear delay impulsive differential equations," Journal of Mathematical Analysis and Applications, vol. 193, no. 3, pp. 923-941, 1995.

[15] A. M. Samorlenko and N. A. Perestyuk, Impulsive Differential Equations, vol. 14 of World Scientific Series on Nonlinear Science. Series A: Monographs and Treatises, World Scientific Publishing, Singapore, 1995.

[16] A. M. Samoilenko and N. A. Perestyuk, Differential Equations with Impulse Effect, Visca Skola, Kiev, Ukraine, 1987.

[17] S. G. Hristova, "Integral stability in terms of two measures for impulsive functional differential equations," Mathematical and Computer Modelling, vol. 51, no. 1-2, pp. 100-108, 2010. 
[18] M. U. Akhmetov and N. A. Perestyuk, "Almost periodic solutions of nonlinear sampled-data systems," Ukrainian Mathematical Journal, vol. 41, no. 3, pp. 259-263, 1989.

[19] M. De la Sen, "Stability of impulsive time-varying systems and compactness of the operators mapping the input space into the state and output spaces," Journal of Mathematical Analysis and Applications, vol. 321, no. 2, pp. 621-650, 2006.

[20] M. De La Sen and N. Luo, "A note on the stability of linear time-delay systems with impulsive inputs," IEEE Transactions on Circuits and Systems I, vol. 50, no. 1, pp. 149-152, 2003.

[21] J. Xu and J. Sun, "Finite-time stability of linear time-varying singular impulsive systems," IET Control Theory E Applications, vol. 4, no. 10, pp. 2239-2244, 2010.

[22] Y. Zhang and J. Sun, "Stability of impulsive linear differential equations with time delay," IEEE Transactions on Circuits and Systems II, vol. 52, no. 10, pp. 701-705, 2005.

[23] X. Fan, F. Jiang, and H. Zhang, "Dynamics of multi-species competition-predator system with impulsive perturbations and Holling type III functional responses," Nonlinear Analysis: Theory, Methods E Applications, vol. 74, no. 10, pp. 3363-3378, 2011.

[24] S. Ahmad and I. M. Stamova, "Asymptotic stability of an N-dimensional impulsive competitive system," Nonlinear Analysis: Real World Applications, vol. 8, no. 2, pp. 654-663, 2007.

[25] X.-z. He and K. Gopalsamy, "Persistence, attractivity, and delay in facultative mutualism," Journal of Mathematical Analysis and Applications, vol. 215, no. 1, pp. 154-173, 1997.

[26] B. S. Goh, "Stability in models of mutualism," The American Naturalist, vol. 113, no. 2, pp. 261-275, 1979.

[27] R. E. Gaines and J. L. Mawhin, Coincidence Degree, and Nonlinear Differential Equations, vol. 568 of Lecture Notes in Mathematics, Springer, Berlin, Germany, 1977.

[28] S. Ahmad and I. M. Stamova, "Asymptotic stability of competitive systems with delays and impulsive perturbations," Journal of Mathematical Analysis and Applications, vol. 334, no. 1, pp. 686-700, 2007. 


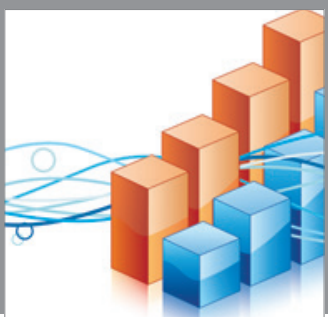

Advances in

Operations Research

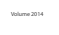

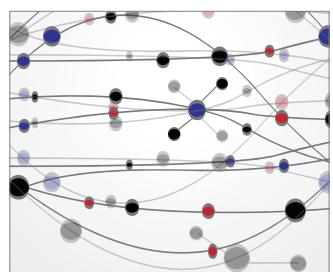

\section{The Scientific} World Journal
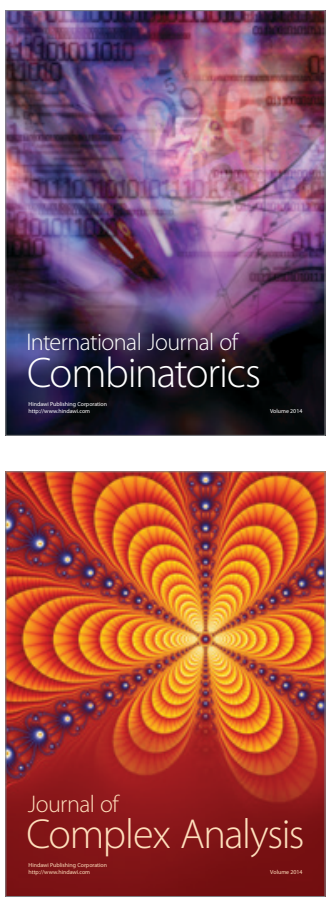

International Journal of

Mathematics and

Mathematical

Sciences
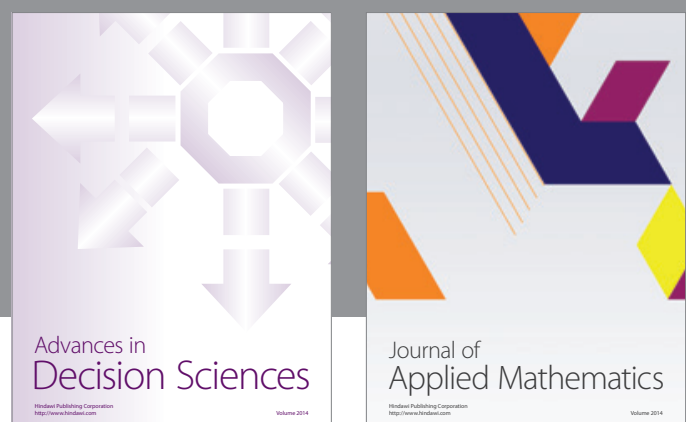

Journal of

Applied Mathematics
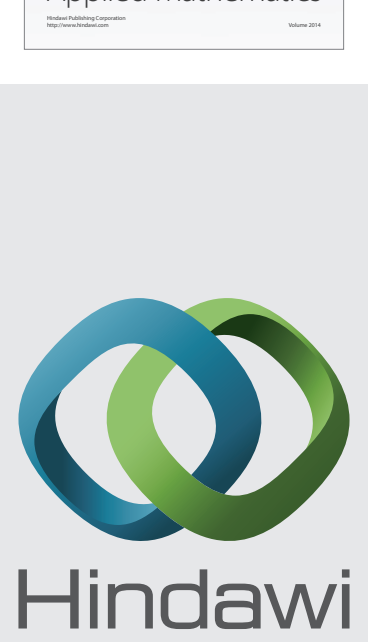

Submit your manuscripts at http://www.hindawi.com
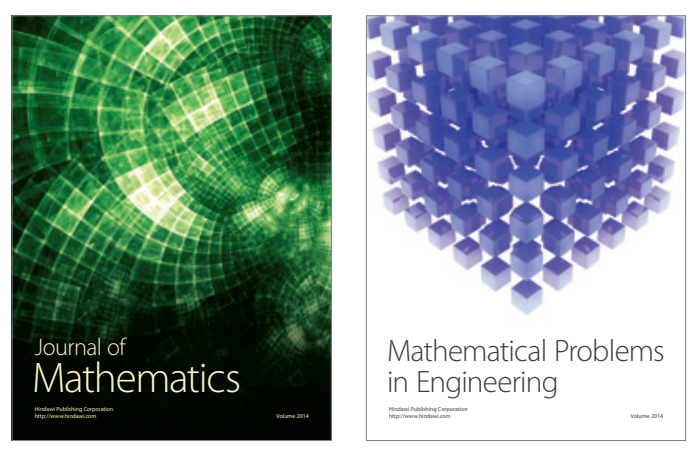

Mathematical Problems in Engineering
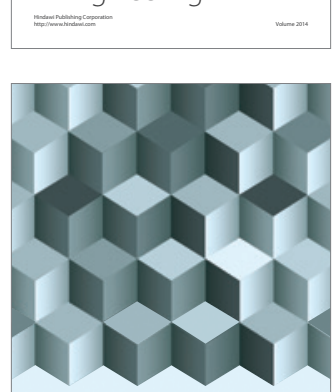

Journal of

Function Spaces
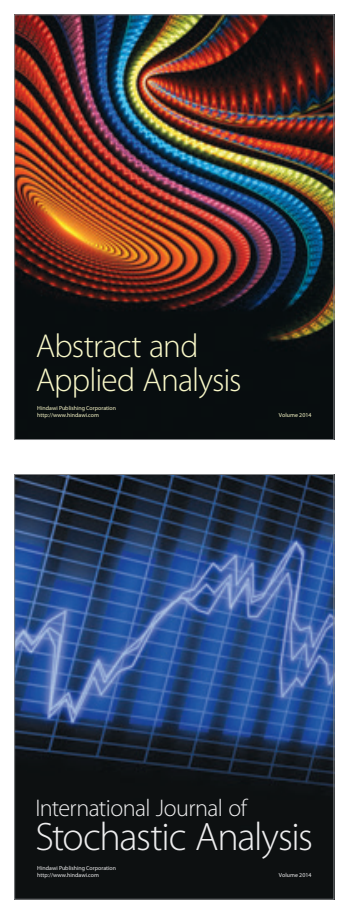

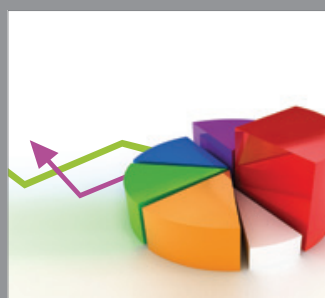

ournal of

Probability and Statistics

Promensencen
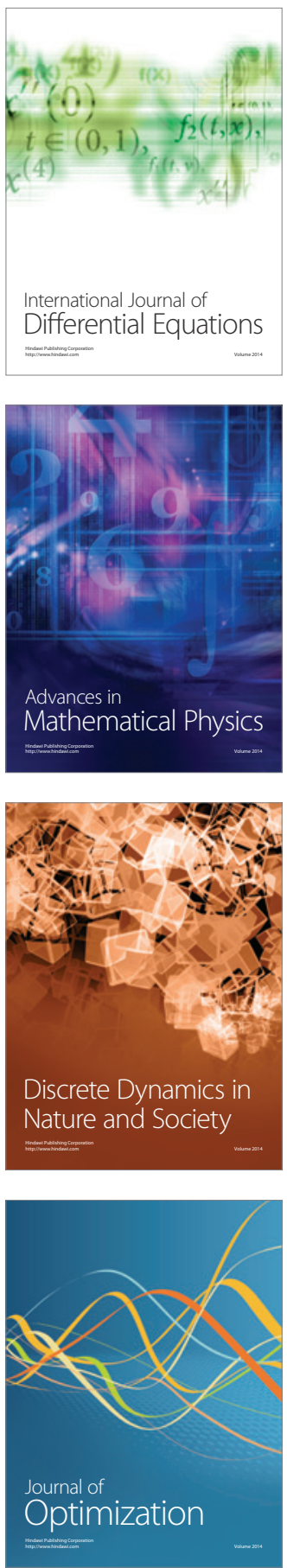